\title{
A Pieri-type formula for even orthogonal Grassmannians
}

\author{
by \\ Piotr Pragacz and Jan Ratajski (Warszawa)
}

Dedicated to Wojtek

\begin{abstract}
We study the cohomology ring of the Grassmannian $G$ of isotropic $n$ subspaces of a complex $2 m$-dimensional vector space, endowed with a nondegenerate orthogonal form (here $1 \leq n<m$ ). We state and prove a formula giving the Schubert class decomposition of the cohomology products in $H^{*}(G)$ of general Schubert classes by "special Schubert classes", i.e. the Chern classes of the dual of the tautological vector bundle of rank $n$ on $G$. We discuss some related properties of reduced decompositions of "barred permutations" with even numbers of bars, and divided differences associated with the even orthogonal group $S O(2 m)$.
\end{abstract}

\section{Contents}

1. Introduction 49

2. Barred permutations and shapes 51

3. Reduced decompositions and divided differences 54

4. Schubert classes and the characteristic map 63

5. When does $\partial_{\mu}^{D}(E)$ vanish? 66

6. Some terminology and the main result 69

7. Proof of the theorem 71

7.1. Case 1: $\lambda$ and $\mu$ are of type 171

7.2. Case $2: \lambda$ is of type 2 and $\mu$ is of type 175

7.3. Case 3: $\lambda$ is of type 1 and $\mu$ is of type $2 \quad 82$

7.4. Case $4: \lambda$ and $\mu$ are of type 289

8. Concluding remarks 94

References 95

1. Introduction. In the present paper, we give a multiplication formula "of Pieri type" for the cohomology rings of the homogeneous spaces $S O(2 m) / P$, where $P$ is a maximal parabolic subgroup of $S O(2 m)$. The paper is a continuation of [PR1] and especially [PR2] where the symplectic and odd orthogonal Pieri-type formulas were given. We refer to [P1, Section 6] for a summary of our method of finding and proving this type of formulas.

2000 Mathematics Subject Classification: 14M15, 05E05.

Research supported by Polish KBN grant No. 2 P03A 02423. 
This method is based on the study of an iterated Leibniz-type formula for divided differences and related deformations of some "distinguished" reduced decompositions of elements of the Weyl group. When reading the present paper the reader should especially invoke the content of [P1, Theorem 6.3] $\left(^{1}\right)$.

Let $G$ be the Grassmannian of isotropic $n$-subspaces of a complex $2 m$ dimensional vector space, endowed with a nondegenerate orthogonal form (here $1 \leq n<m$ ). To formulate our Pieri-type formula, in Section 4 we specify some $n+1$ special Schubert classes which generate multiplicatively the cohomology ring of $G$. (In fact, these are the Chern classes of the dual of the tautological rank $n$ vector bundle on $G$ together with a certain element from $H^{2(m-n)}(G)$.) We then discuss the Schubert class decomposition of the product of a general Schubert class by a special one. The idea of the proof of the main result is similar to that in [PR1], [PR2], and [DP], and several results from these papers can be directly applied here. The work with even orthogonal groups requires a different and more involved combinatorics related to reduced decompositions than in the symplectic and odd orthogonal cases. Our method calculates explicitly the non-zero summands in the Pieri type products together with their multiplicities. The core of the method is to show that the sum in Proposition 4.6 contains at most one non-zero term. We then analyze necessary and sufficient conditions for the occurrence of such a term.

Here is an outline of the content of the paper.

Sections 1-4 contain basic information about the Weyl group of type $D_{m}$, and some combinatorial objects called "shapes" indexing Schubert classes in the cohomology of even orthogonal Grassmannians. Reduced decompositions of barred permutations corresponding to shapes are investigated, and a description of the cohomology rings of such Grassmannians is given in terms of symmetric polynomials; this leads to a quick derivation of a "compact" expression for the Poincaré series of these varieties. Especially important is the description of the Borel characteristic map with the help of divided differences due to Bernstein, I. M. Gelfand and S. I. Gelfand [BGG], and Demazure [D1], [D2]. In order to compute with the iterated Leibniz-type formula for products of divided differences, we consider some compositions of simple reflections and divided differences. (In the $S L(m)$ case, such an "algebra of divided differences" has recently been extensively studied by Lascoux and Schützenberger [LS1]-[LS3].)

In Section 5, we provide certain explicit criterions for the vanishing of these operators when applied to some generating functions.

In Section 6, we introduce some terminology and state the main result, Theorem 6.2.

$\left({ }^{1}\right)$ In $[\mathrm{P} 2]$ we show how our method reproves the classical Pieri formula [Pi] for the Grassmannians $S L(m) / P$, where $P$ is a maximal parabolic subgroup of $S L(m)$. 
In Section 7 , we prove Theorem 6.2 by checking four separate cases. In the proof we invoke several results from [PR2] and [DP], and give detailed arguments for all new assertions.

In this paper, we deal only with the Grassmannians of non-maximal isotropic subspaces. A group-theoretic treatment of a Pieri-type formula in the maximal case was given in [DP]. Note that the case $n=1$, when $G$ is a quadric hypersurface in $\mathbb{P}^{2 m-1}$, is well known (cf. Example 4.4 and Remark 7.41). It seems that Corrado Segre was the first to describe, 120 years ago in his "dissertazione di laurea" [Se], what we call today the "cohomology ring" of such a quadric. The present paper gives a Pieri-type formula for the cohomology ring of the variety of $(n-1)$-spaces, lying on a smooth $(2 m-2)$-dimensional quadric.

In [Exx] we collect, for the reader's convenience, several examples illustrating various operations performed in this paper. (In particular, these examples show that no transformation of the distinguished reduced decomposition studied in the present paper in order to get the unique $D$ from Proposition 4.6 can be omitted.)

For other approaches to cohomology Pieri-type formulas for various Grassmannians, see the papers: [HB] by Hiller and Boe, [S] by Sertöz, and [So2], [So3] by Sottile. The first paper is based on Chevalley's multiplication formula $[\mathrm{C}]$, the remaining ones use linear algebra methods. We also mention the existence of other cohomology "Pieri formulas" generalizing the Monk formula for flag varieties by Lascoux and Schützenberger [LS1], Sottile [So1], and Veigneau $[\mathrm{V}]$.

For an indication of a possible application of the present paper to quantum cohomology, see the paper [BKT] by Buch, Kresch and Tamvakis.

This paper is a substantially revised and expanded version of our preprint [PR3].

2. Barred permutations and shapes. Let $H=S O(2 m)$ be the orthogonal group (of type $D_{m}$ ) over the field of complex numbers. The following notation will be used throughout the paper: $B$ is a fixed Borel subgroup of $H ; T \subset B$ a fixed maximal torus; $\mathcal{R}$ the root system of $H$ associated with $T ; \Sigma$ a set of simple roots of $\mathcal{R}$ associated with $B$; and $W$ the Weyl group of $(H, T)$. In a standard realization from [Bou]:

$$
\begin{aligned}
& \mathcal{R}=\left\{ \pm \varepsilon_{i} \pm \varepsilon_{j} \mid 1 \leq i<j \leq m\right\} \subset \mathbb{R}^{m}=\bigoplus_{i=1}^{m} \mathbb{R} \varepsilon_{i}, \\
& \Sigma=\left\{\varepsilon_{1}-\varepsilon_{2}, \ldots, \varepsilon_{m-1}-\varepsilon_{m}, \varepsilon_{m-1}+\varepsilon_{m}\right\}, \quad W=S_{m} \ltimes \mathbb{Z}_{2}^{m-1} .
\end{aligned}
$$

A typical element of $W$ can be written as a pair $(\tau, \epsilon)$, where $\tau \in S_{m}$ and $\epsilon=\left(\epsilon_{1}, \ldots, \epsilon_{m}\right)$ is a sequence of elements of $\mathbb{Z}_{2}=\{-1,1\}$ such that 
$\#\left\{i \mid \epsilon_{i}=-1\right\}$ is even. Multiplication in $W$ is given by $(\tau, \epsilon) \cdot\left(\tau^{\prime}, \epsilon^{\prime}\right)=$ $\left(\tau \circ \tau^{\prime}, \delta\right)$, where "०" denotes the composition of permutations and $\delta_{i}=$ $\epsilon_{\tau^{\prime}(i)} \cdot \epsilon_{i}^{\prime}$. For elements in $W$, we shall use the "barred permutation" notation, indicating by bars the places in the permutation $w=[w(1), \ldots, w(m)]=$ $w(1) w(2) \ldots w(m)$ where $\epsilon_{i}=-1$. The simple reflections corresponding to simple roots from $\Sigma$ are $s_{i}=[1, \ldots, i-1, i+1, i, i+2, \ldots, m], i=1, \ldots, m-1$, and $s_{m}=[1, \ldots, m-2, \bar{m}, \overline{m-1}]$.

The length function on the group $W$ relative to $\Sigma$ is given by

$$
l(w)=\sum_{i=1}^{m} a_{i}+\sum_{\epsilon_{j}=-1} 2 b_{j},
$$

where $a_{i}=\#\{j \mid j>i \& w(j)<w(i)\}$ and $b_{j}=\#\{i \mid i>j \& w(i)>$ $w(j)\}$.

For $1 \leq n<m$, let $W_{n}$ be the subgroup of $W$ generated by the simple reflections $s_{i}$, where $i \neq n$. Note that $W_{n} \simeq S_{n} \times\left(S_{m-n} \ltimes \mathbb{Z}_{2}^{m-n-1}\right)$. (If $m=n+1$ then $W_{n} \simeq S_{n} \times S_{1}$.)

Consider the poset $W^{(n)}$ of the minimal length left coset representatives of $W_{n}$ in $W$. This poset decomposes into two disjoint subsets $W^{(n)}=W_{1}^{(n)} \dot{U}$ $W_{2}^{(n)}$, where

$$
\begin{aligned}
& W_{1}^{(n)}=\left\{\left[y_{1}, y_{2}, \ldots, y_{n-k} ; \bar{z}_{k}, \bar{z}_{k-1}, \ldots, \bar{z}_{1} ; v_{1}, \ldots, v_{m-n}\right] \mid k \text { even }\right\}, \\
& W_{2}^{(n)}=\left\{\left[y_{1}, y_{2}, \ldots, y_{n-k} ; \bar{z}_{k}, \bar{z}_{k-1}, \ldots, \bar{z}_{1} ; v_{1}, \ldots, v_{m-n-1}, \bar{v}_{m-n}\right] \mid k \text { odd }\right\},
\end{aligned}
$$

where the $y$ 's, $z$ 's, and $v$ 's are integers, and $y_{1}<\ldots<y_{n-k}, z_{k}>\ldots>z_{1}$, $v_{1}<\ldots<v_{m-n}$ in both instances. We refer to the elements of these subsets as permutations of type 1 and of type 2 respectively.

We set $\alpha=m-n$, and $\beta=m-n-1$ if this last number is positive.

In this paper, any unexplained terminology related to partitions, Ferrers diagrams etc. is borrowed from $[\mathrm{M}]$; sometimes, however, we use different notation.

Besides the usual length $l(\lambda)$ of a partition $\lambda$, we shall also use $i(\mu)$ : for a partition $\mu$ with $l=l(\mu)$ even, we set

$$
\stackrel{i}{l}(\mu)= \begin{cases}l & \text { if } \mu_{l}>1 \\ l-1 & \text { if } \mu_{l}=1 .\end{cases}
$$

Definition 2.1. Let $\lambda=\left(\lambda^{t} / / \lambda^{b}\right)$ be a pair of strict partitions $\lambda^{t}$ and $\lambda^{b}$ such that $\lambda^{t} \subset\left(m^{\alpha}\right), \lambda^{b} \subset\left(m^{n}\right)$ for $n$ even, $\lambda^{b} \subset\left(m^{n}, 1\right)$ for $n$ odd, and $l\left(\lambda^{b}\right)$ is even. Then $\lambda$ is called:

(i) a shape of type 1 if $\lambda_{\alpha}^{t} \geq l\left(\lambda^{b}\right)+1$;

(ii) a shape of type 2 if $\lambda_{\alpha}^{t} \leq l\left(\lambda^{b}\right), \lambda_{\beta}^{t} \geq \stackrel{i}{l}\left(\lambda^{b}\right)+2$, and $\lambda_{\alpha}^{t}+\lambda_{s}^{b} \leq \lambda_{\beta}^{t}$ for $s=\lambda_{\alpha}^{t}$. 
It will be useful to picture shapes as sets of boxes in the plane. Let $D_{\lambda}^{t}$ and $D_{\lambda}^{b}$ denote the Ferrers diagrams of $\lambda^{t}$ and $\lambda^{b}$. The diagram $D_{\lambda}$ of the shape $\left(\lambda^{t} / / \lambda^{b}\right)$ is the juxtaposition of $D_{\lambda}^{t}$ and $D_{\lambda}^{b}$, and has rows with successive lengths: $\lambda_{1}^{t}, \ldots, \lambda_{\alpha}^{t} ; \lambda_{1}^{b}, \ldots, \lambda_{l}^{b}$, where $l=l\left(\lambda^{b}\right)$. We depict the shapes $(9,7,6 / / 7,6,4,1),(7,5,3 / / 8,7,2,1)$, and $(7,6,3 / / 8,7,3,2)$ (here we take $m \geq 9)$ :

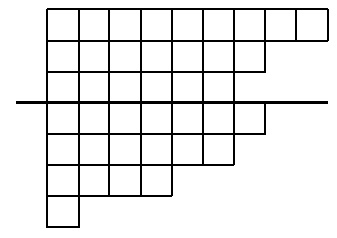

type 1
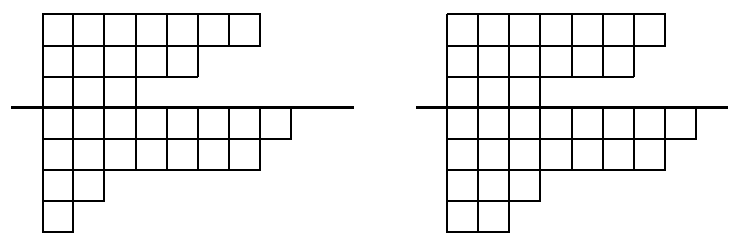

type 2

Sometimes we shall use dots, circles or asterisks to indicate the boxes of a shape.

We now discuss bijections between the sets $W_{i}^{(n)}$ of permutations $(i=$ $1,2)$ and the sets of shapes of type 1 and 2 . For a given $w \in W^{(n)}$, we define the corresponding shape $\lambda=\left(\lambda^{t} / / \lambda^{b}\right)$ in the following way. First, for $r=1, \ldots, \alpha$, we set $d_{r}:=\#\left\{z_{j}<v_{r}\right\}$.

- If $w=\left[y_{1}, \ldots, y_{n-k} ; \bar{z}_{k}, \bar{z}_{k-1}, \ldots, \bar{z}_{1} ; v_{1}, \ldots, v_{\alpha}\right]$ is of type 1 , then we put

$$
\begin{array}{ll}
\lambda_{j}^{b}=m+1-z_{j} & \text { for } j=1, \ldots, k \\
\lambda_{r}^{t}=m+1-v_{r}+d_{r} & \text { for } r=1, \ldots, \alpha .
\end{array}
$$

- For $w=\left[y_{1}, \ldots, y_{n-k} ; \bar{z}_{k}, \bar{z}_{k-1}, \ldots, \bar{z}_{1} ; v_{1}, \ldots, \bar{v}_{\alpha}\right]$ of type 2 , the parts of the strict partition $\lambda^{b}=\left(\lambda_{1}^{b}>\ldots>\lambda_{k+1}^{b}\right)$ are elements of the set

$$
\left\{m+1-z_{1}, \ldots, m+1-z_{k}, m+1-v_{\alpha}\right\} .
$$

For $r=1, \ldots, \beta$, we set $\lambda_{r}^{t}=m+1-v_{r}+d_{r}$, and we put $\lambda_{\alpha}^{t}=1+d_{\alpha}$.

LEMma 2.2. The assignment described above gives a bijection between $W_{1}^{(n)}\left(\right.$ resp. $\left.W_{2}^{(n)}\right)$ and the set of shapes of type 1 (resp. 2).

Proof. Type 1: The resulting $\lambda^{b}$ and $\lambda^{t}$ are strict partitions (for the latter we note that $\left(v_{i}-d_{i}\right)$ is strictly increasing). These partitions are bounded as in Definition 2.1. The inequality $\lambda_{\alpha}^{t} \geq l\left(\lambda^{b}\right)+1$ is checked as in the proof of [PR2, Lemma 1.2]. Also the converse assignment: "shape $\rightarrow$ permutation" may be given in the same way as in that proof. But it is easier to see it using the graphical presentation of shapes, as will be explained after Example 3.3. 
Type 2: The $\lambda^{b}$ is a strict partition. Since $\left(v_{i}-d_{i}\right)$ is strictly increasing, we have

$$
\lambda_{1}^{t}>\ldots>\lambda_{\beta}^{t}=m+1-v_{\beta}+d_{\beta} \geq\left(1+d_{\alpha}\right)+\left(m+1-v_{\alpha}\right)=\lambda_{\alpha}^{t}+\lambda_{s}^{t},
$$

where $s=\lambda_{\alpha}^{t}$.

The strict partitions are bounded as in Definition 2.1. The inequality $\lambda_{\beta}^{t} \geq \stackrel{i}{l}\left(\lambda^{b}\right)+2$ for $\stackrel{i}{l}\left(\lambda^{b}\right)=l\left(\lambda^{b}\right)$ is equivalent to $v_{\beta} \leq m-2-\left(k-d_{\beta}\right)$, and it holds because $v_{\alpha}$ and $m$ (which is neither $v_{\alpha}$ nor any $z$ ) are greater than $v_{\beta}$. If $l\left(\lambda^{b}\right)=l\left(\lambda^{b}\right)-1$, the inequality is equivalent to $v_{\beta} \leq m-1-\left(k-d_{\beta}\right)$ and it holds because $v_{\alpha}>v_{\beta}$. Also, we have $\lambda_{\alpha}^{t} \leq 1+k=l\left(\lambda^{b}\right)$.

The converse assignment: "shape $\rightarrow$ permutation" is best seen using the graphical presentation of shapes, and will be explained after Example 3.3.

EXAmple 2.3. Here $m=8 ;(7,5 / / 8,6,4,1)$ corresponds to $[2,6 ; \overline{8}, \overline{5}, \overline{3}, \overline{1}$; $4,7],(8,6,3 / / 8,5,3,2)$ corresponds to $[3,8 ; \overline{7}, \overline{4}, \overline{1} ; 2,5, \overline{6}]$, and $(8,5,3 / / 8,5$, $2,1)$ corresponds to $[3,5 ; \overline{8}, \overline{4}, \overline{1} ; 2,6, \overline{7}]$.

3. Reduced decompositions and divided differences. Fix $w \in$ $W^{(n)}$. In this section we deal with a given reduced decomposition $w=$ $s_{i_{1}} \cdots s_{i_{l}}$ as a sequence of simple transposition operations which produces $w$ from the identity permutation:

$$
w=\left(\cdots\left([1,2, \ldots, m] \cdot s_{i_{1}}\right) \cdots\right) \cdot s_{i_{l}} .
$$

In the following, the simple transpositions involved will be called the $s_{i_{h}}$ operations $(h=1, \ldots, l)$, or s-operations.

Remembering that some elements receive bars, we omit the bars in the following lemma, for simplicity of exposition.

Lemma 3.1. The s-operations transpose exactly the following pairs. Each pair $\left(z_{i}<v_{j}\right)$ is transposed twice: $\left(z_{i}, v_{j}\right) \rightarrow\left(v_{j}, z_{i}\right) \rightarrow\left(z_{i}, v_{j}\right)$, while the pairs $\left(v_{j}<z_{i}\right),\left(z_{i}<z_{j}\right),\left(z_{j}<y_{i}\right)$ and $\left(v_{i}<y_{j}\right)$ are each transposed once.

Proof. It is clear that we must at least make the indicated transpositions. For example, we have to transpose each pair $\left(z_{i}, v_{j}\right)$ where $z_{i}<v_{j}$ at least twice because $z_{i}$ must receive a bar and $z_{i}$ precedes $v_{j}$ in $w$; also each pair $\left(z_{i}, z_{j}\right)$ where $i<j$ must be transposed at least once because in $w$ we have the ordering $z_{k}, \ldots, z_{1}$. One argues in a similar way for the necessity of the other transpositions. In total we need at least

$$
\begin{aligned}
2 \sum \#\left\{\left(z_{i}<v_{j}\right)\right\}+\sum \#\left\{\left(z_{i}>v_{j}\right)\right\} & +\sum \#\left\{\left(z_{i}>z_{j}\right)\right\} \\
& +\sum \#\left\{\left(y_{i}>z_{j}\right)\right\}+\sum \#\left\{\left(y_{j}>v_{i}\right)\right\}
\end{aligned}
$$

$s$-operations. But this sum is equal to $l(w)$ by formula (1). Note that the first sum in the above expression is $2 \sum b$, and the remaining sums give $\sum a$ in (1). This proves the lemma. 
In the following proposition, we also take into account bars. Moreover, $[\ldots, a, b, \ldots] \rightarrow[\ldots, b, a, \ldots]$ will denote an $s_{i_{h}}$-operation also with $i_{h}<m$, and $[\ldots, a, b] \rightarrow[\ldots, \bar{b}, \bar{a}]$ will be the $s_{m}$-operation. (Note that the $s_{m-1^{-}}$ operation also acts on the two righmost components, but without bars, and so it will be denoted by the former symbol.)

Proposition 3.2. Any s-operation belongs to one of the following types:

(i) $\left[\ldots, z_{i}, x, \ldots\right] \rightarrow\left[\ldots, x, z_{i}, \ldots\right]$, where $x \neq z_{j}$;

(ii) $\left[\ldots, v_{i}, x, \ldots\right] \rightarrow\left[\ldots, x, v_{i}, \ldots\right]$, where $x$ is some $y_{j}$ or $\bar{z}_{j}$;

(iii) $[\ldots, a, b] \rightarrow[\ldots, \bar{b}, \bar{a}]$, where $(a, b)$ can be one of the pairs:

$$
\left(z_{i}, z_{i+1}\right),\left(z_{i}, v_{\alpha}\right),\left(v_{\alpha}, z_{i}\right),\left(\bar{v}_{\alpha}, z_{i}\right),\left(z_{i}, \bar{v}_{\alpha}\right)
$$

(iv) $\left[\ldots, \bar{v}_{\alpha}, \bar{z}_{i}, \ldots\right] \rightarrow\left[\ldots, \bar{z}_{i}, \bar{v}_{\alpha}, \ldots\right]$.

Proof. In the proof, we shall repeatedly use the previous lemma.

Type 1: First, looking at the list of transpositions in the lemma, we see that each of them is one of the $s$-operations (i)-(iv). Now we shall justify the described properties of the $s$-operations (i)-(iv).

Since a pair $\left(z_{i}<z_{j}\right)$ is transposed once, each such transposition is of the form $\left[\ldots, z_{i}, \bar{z}_{j}, \ldots\right] \rightarrow\left[\ldots, \bar{z}_{j}, z_{i}, \ldots\right]$. Any other $s$-operation moving $z_{i}$ forward is possible. This is what (i) says.

Among the $s$-operations, there is no transposition between two $v$ 's, and any transposition corresponding to $\left(v_{i}<z_{j}\right)$ must be of the form $\left[\ldots, v_{i}\right.$, $\left.\bar{z}_{j}, \ldots\right] \rightarrow\left[\ldots, \bar{z}_{j}, v_{i}, \ldots\right]$. Any element $v_{i}$ on the way to its own place in $w$ can be transposed only with $y$ 's or with $\bar{z}$ 's, as asserted in (ii).

There is no transposition between two $v$ 's; hence among the $v$ 's only $v_{\alpha}$ can receive a bar (and then must lose it). There is no transposition between two $y$ 's, and any $y_{i}$ can only be moved backward via an $s$-operation. It follows that no $y$ can receive a bar. Hence every $s_{m}$-operation is of the form described in (iii): only $z$ 's and $v_{\alpha}$ can receive bars.

If $v_{\alpha}$ receives a bar, then it must lose it. (In fact, this can happen several times.) The element $\bar{v}_{\alpha}$ on its way toward the $m$ th place cannot be interchanged with $v_{i}$ (there is no transposition between two $v$ 's), with $y_{i}$ (such a transposition can occur before $v_{\alpha}$ is supplied with a bar), and with a bar-free $z_{i}$. This is what (iv) says.

Type 2: We discuss the bars that appear or disappear under the sequence of $s$-operations. As in type 1 , every $z$ receives a bar and does not lose it. This follows from the form of the transpositions between $z$ 's and $v$ 's among the $s$-operations.

Among the $v$ 's, only $v_{\alpha}$ receives a bar because no transposition between the $v$ 's occurs. It can happen that $v_{\alpha}$ receives a bar, then loses it, and receives it once again. (In fact, this can happen several times.) 
Since any $s$-operation moves $y_{i}$ backward, no $y_{i}$ can be supplied with a bar.

This exhausts all the possibilities when a bar can appear or disappear. A discussion of possibilities (i)-(iv) is similar to Type 1 above.

EXAmPle 3.3. Type $2 ; v_{\alpha}$ receives a bar, then loses it, and then receives a bar again: $12345 \rightarrow 12435 \rightarrow 124 \overline{53} \rightarrow 14 \overline{3} 2 \overline{5} \rightarrow 14 \overline{3} 5 \overline{2} \rightarrow 14 \overline{32} 5 \rightarrow$ $4 \overline{32} 15 \rightarrow 4 \overline{3251} \rightarrow \overline{321} 4 \overline{5}$.

Type $1 ; v_{\alpha}$ receives a bar twice and loses it twice: $12345 \rightarrow 123 \overline{54} \rightarrow$ $\overline{4} 123 \overline{5} \rightarrow \overline{4} 125 \overline{3} \rightarrow \overline{4} \overline{3} 125 \rightarrow \overline{43} 1 \overline{52} \rightarrow \overline{432} 1 \overline{5} \rightarrow \overline{432} 5 \overline{1} \rightarrow \overline{4321} 5$.

Type $2 ; v_{\alpha}$ receives a bar 3 times and loses it twice: $123456 \rightarrow 1234 \overline{65} \rightarrow$ $\overline{5} 1234 \overline{6} \rightarrow \overline{5} 1236 \overline{4} \rightarrow \overline{54} 1236 \rightarrow \overline{54} 12 \overline{63} \rightarrow \overline{543} 12 \overline{6} \rightarrow \overline{5} \overline{4} 16 \overline{2} \rightarrow \overline{5432} 16 \rightarrow$ $\overline{543261} \rightarrow \overline{543216 .}$

Type 2 ; illustration for (iv): $123456 \rightarrow 1234 \overline{65} \rightarrow \overline{65} 1234 \rightarrow \overline{65} 12 \overline{43} \rightarrow$ $\overline{654312} \rightarrow \overline{654321} \rightarrow \overline{543216 .}$

Suppose a shape $\mu=\left(\mu^{t} / / \mu^{b}\right)$ is given. We now explain how one can read off, from the picture of the shape, the barred permutation which corresponds to it. Let us use the following (matrix) coordinates for boxes in $D_{\mu}^{t}$ and $D_{\mu}^{b}$ :

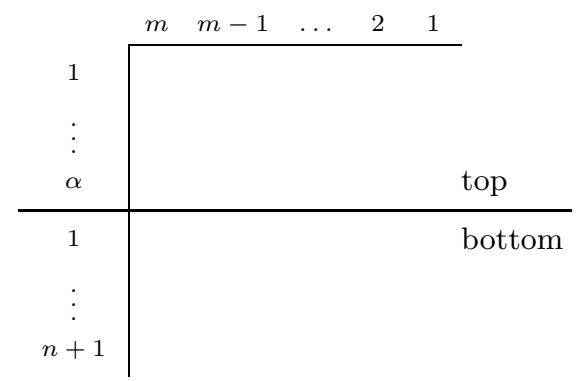

Let us modify the diagram $D_{\mu}$ in the following way.

- Remove from $D_{\mu}^{t}$ the following set of boxes:

$(1, m), \ldots,(1, n+2),(1, n+1)$;

$(2, m), \ldots,(2, n+2)$;

...

$(\alpha, m)$.

- Remove one box from each row of $D_{\mu}^{b}$ : from each even-numbered row remove the box in column $m$, and from each odd-numbered row remove the box in column $m-1$.

We picture the removed boxes as " $\times$ " and denote the resulting set of boxes by $\stackrel{\circ}{D}_{\mu}$. The top (resp. bottom) part of $\stackrel{\circ}{D}_{\mu}$ will be denoted by $\stackrel{\circ}{D}_{\mu}^{t}$ (resp. $\stackrel{\circ}{D}_{\mu}^{b}$ ). 
For example, take the shape $(8,5,3 / / 8,5,2,1)$ and $m=8$ :

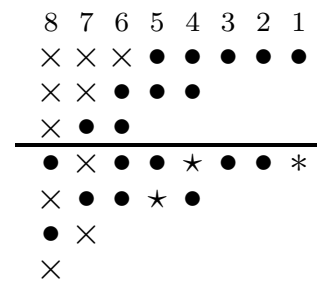

We read the numbers of columns of the boxes without " $x$ " row by row from right to left and from bottom to top, getting a sequence $i_{1}, \ldots, i_{l}$. In our example, we obtain: $8,4,5,6,7,1,2,3,4,5,6,8,6,7,4,5,6,1,2,3,4,5$. The barred permutation corresponding to $\mu$, denoted by $w_{\mu}$, is the composition $s_{i_{1}} \cdots s_{i_{l}}$. In our example, it is

$s_{8} \cdot s_{4} \cdot s_{5} \cdot s_{6} \cdot s_{7} \cdot s_{1} \cdot s_{2} \cdot s_{3} \cdot s_{4} \cdot s_{5} \cdot s_{6} \cdot s_{8} \cdot s_{6} \cdot s_{7} \cdot s_{4} \cdot s_{5} \cdot s_{6} \cdot s_{1} \cdot s_{2} \cdot s_{3} \cdot s_{4} \cdot s_{5}$, which gives the barred permutation $[3,5 ; \overline{8}, \overline{4}, \overline{1}, 2,6, \overline{7}]$. The reduced decomposition just described is the distinguished reduced decomposition of $w_{\mu}$.

We can also read off this permutation from the picture in the following way. The $z$ 's and $v_{\alpha}$ are the elements of the set obtained by subtracting the parts of $\mu^{b}$ from $m+1$, and $v_{\alpha}$ is the $\mu_{\alpha}$ th element in this set put in ascending order. In our example, $\alpha=3,\left\{z_{1}, z_{2}, z_{3}, v_{\alpha}\right\}=\{1,4,7,8\}$, and $v_{\alpha}=7$ because $d_{\alpha}=2$. The remaining $v$ 's are known once we know the $d_{i}$, $i=1, \ldots, \beta$. But $d_{i}$ is the number of boxes in the antidiagonal segment in the bottom part starting in the column of the rightmost box of the $i$ th top row. In our example $d_{2}=2$, which is the number of $\star$ 's, and $d_{1}=1$, which is the number of $*$ 's. Thus, $v_{2}=6$ and $v_{1}=2$. Observe that the inequality $\mu_{3}^{b}+\mu_{3}^{t} \leq \mu_{2}^{t}$ implies that the box $(3,6)$ does not belong to $D_{\mu}^{b}$, which in turn implies that $v_{\beta}<v_{\alpha}$. (In general, it is the inequality $\mu_{\alpha}^{t}+\mu_{s}^{b} \leq \mu_{\beta}^{t}$, with $s=\mu_{\alpha}^{t}$, which ensures that $v_{\beta}<v_{\alpha}$.) Our example is of type 2. Similarly one can proceed with a shape of type 1 , where the $z$ 's are obtained by subtracting the parts of $\mu^{b}$ from $m+1$, and the $d_{i}$ 's are read off from the graphical presentation of the shape in the same way as above.

REMARK 3.4. One has $l\left(w_{\mu}\right)=\# \stackrel{\circ}{D}_{\mu}$ and the maximal length of $w_{\mu}$ is $n(m-n)+(m-1)+(m-2)+\cdots+(m-n)=n(2 m-n)-n(n+1) / 2$.

Besides barred permutations, we shall also need divided differences of type $D_{m}$. Let us first define (simple) divided differences, $\partial_{i}: \mathbb{Z}[X] \rightarrow \mathbb{Z}[X]$, $i=1, \ldots, m$, where $X=\left\{x_{1}, \ldots, x_{m}\right\}$ is a fixed set of indeterminates, by setting

$$
\begin{aligned}
\partial_{i}(f) & =\left(f-s_{i} f\right) /\left(x_{i}-x_{i+1}\right), \quad i=1, \ldots, m-1, \\
\partial_{m}(f) & =\left(f-s_{m} f\right) /\left(x_{m-1}+x_{m}\right) .
\end{aligned}
$$


The transposition $s_{i}, i=1, \ldots, m-1$, acts on $\mathbb{Z}[X]$ by interchanging $x_{i}$ and $x_{i+1}$, and $s_{m}$ acts by transposing $x_{m-1}$ with $x_{m}$ and multiplying both variables by -1 (the remaining variables are invariant). Since simple divided differences satisfy the Coxeter relations with the $s_{i}$ 's, with any $w \in W$ there is associated a divided difference $\partial_{w}: \mathbb{Z}[X] \rightarrow \mathbb{Z}[X]$ defined as $\partial_{i_{1}} \circ \cdots \circ \partial_{i_{l}}$ for any reduced decomposition $w=s_{i_{1}} \cdots s_{i_{l}}$.

Suppose now that a subset $D \subset \stackrel{\circ}{D}_{\mu}$ is given. A box belonging to $D$ will be called a $D$-box, and a box in $\stackrel{\circ}{D}_{\mu} \backslash D$ will be a $\sim D$-box. A $D$-box will be pictured as "•", and a $\sim D$-box as "o" or, if there is no danger of confusion, as a white box. By $D^{t}$ (resp. $D^{b}$ ) we shall denote the restriction of $D$ to $D_{\mu}^{t}$ (resp. to $D_{\mu}^{b}$ ).

The operators $\partial_{\mu}^{D}: \mathbb{Z}[X] \rightarrow \mathbb{Z}[X]$ defined in the next definition will be of particular importance in the present paper.

Definition 3.5. Read $\stackrel{\circ}{D}_{\mu}$ row by row from left to right and from top to bottom. Every $D$-box (resp. $\sim D$-box) in column $i$ gives us $s_{i}$ (resp. $\partial_{i}$ ). Then $\partial_{\mu}^{D}$ is the composition of the resulting $s_{i}$ 's and $\partial_{i}$ 's (the composition written from right to left), and $r_{D}$ is the word obtaining by erasing all the $\partial_{i}$ 's from $\partial_{\mu}^{D}$.

For example,

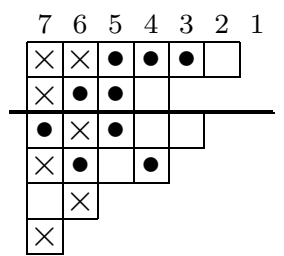

$$
\begin{aligned}
& m=7, n=5 \\
& \mu=(6,4 / / 5,4,2,1)
\end{aligned}
$$

$\partial_{\mu}^{D}=\partial_{7} \circ s_{4} \circ \partial_{5} \circ s_{6} \circ \partial_{3} \circ \partial_{4} \circ s_{5} \circ s_{7} \circ \partial_{4} \circ s_{5} \circ s_{6} \circ \partial_{2} \circ s_{3} \circ s_{4} \circ s_{5}$,

$r_{D}=s_{4} \cdot s_{6} \cdot s_{5} \cdot s_{7} \cdot s_{5} \cdot s_{6} \cdot s_{3} \cdot s_{4} \cdot s_{5}$.

We see that if $D=\emptyset$ then $\partial_{\mu}^{D}=\partial_{w_{\mu}}$, and if $D=\stackrel{\circ}{D}_{\mu}$ then $r_{D}$ is exactly our distinguished reduced decomposition of $w_{\mu}$.

Suppose now that another shape $\lambda$ is given, and that $D \subset \stackrel{\circ}{D}_{\mu}$ is such that $r_{D} \in R\left(w_{\lambda}\right)\left({ }^{2}\right)$. The $D$-boxes correspond to $s$-operations appearing in $r_{D}$. Proposition 3.2 allows us to define the notion of a mark of a $D$-box. We shall say that a $D$-box $\mathfrak{a}$ has $z$-mark (resp. $v$-mark) $j$ if $z_{j}$ (resp. $v_{j}$ ) is non-trivially involved in the $s$-operation associated with $\mathfrak{a}$. (Note that a box in column $m$ can have a double $z$-mark.) Also, we say that a $D$-box $\mathfrak{a}$ has $\bar{v}$-mark if $\bar{v}_{\alpha}$ is non-trivially involved in the $s$-operation corresponding to $\mathfrak{a}$.

$\left(^{2}\right)$ For a given $w \in W$, we denote by $R(w)$ the set of its reduced decompositions. 
A box is called a $z$-box if the corresponding $s$-operation moves some $z_{j}$ forward. A box is called a $v$-box (resp. $\bar{v}$-box) if it has some $v$-mark (resp. $\bar{v}$-mark). We say that a box $\mathfrak{a}$ is a pure $v$-box with $v$-mark $j$ (resp. a pure $\bar{v}$-box) if the associated $s$-operation moves $v_{j}\left(\right.$ resp. $\left.\bar{v}_{\alpha}\right)$ forward (toward the $m$ th place).

Definition 3.6. A $z$-ribbon (resp. v-ribbon) with mark $j$ is the set of all $D$-boxes whose $z$-marks (resp. $v$-marks) are $j$. A $v$-ribbon with mark $\alpha$ will also be called the $v_{\alpha}$-ribbon. The $\bar{v}$-ribbon is the set of all $D$-boxes with a $\bar{v}$-mark. The union of the $v_{\alpha}$-ribbon and the $\bar{v}$-ribbon will be called the $\bar{v} / v$-ribbon.

As a consequence of Proposition 3.2, we get the following properties of ribbons.

Proposition 3.7. (i) (Connectedness) The z-boxes, pure v-boxes and pure $\bar{v}$-boxes with a fixed mark form connected sets in each row, i.e. their column numbers form an interval in $\{m, m-2, m-1, \ldots, 1\}$ (resp. in $\{m-1$, $m-2, \ldots, 1\})$ for an odd-numbered (resp. even-numbered) row.

(ii) (Separation) In a fixed row, two sets of D-boxes are separated (i.e. there is at least one $\sim D$-box between them) provided:

- they are equipped with two different $z$-marks;

- they are pure v-boxes (or $\bar{v}$-boxes) equipped with two different marks;

- one of them consists of z-boxes with a fixed mark and the second of pure $v$-or $\bar{v}$-boxes with a fixed mark.

(iii) The $z$-ribbon with a fixed mark $j$ is contained entirely in the bottom part of $D_{\mu}$. For $j$ odd, it is of the form

$$
\left(t_{m}, m\right),\left(t_{m-2}, m-2\right),\left(t_{m-3}, m-3\right), \ldots,\left(t_{z_{j}}, z_{j}\right),
$$

where $j \leq t_{m} \leq t_{m-2} \leq \ldots \leq t_{z_{j}}$. For $j$ even, it is of the form

$$
\left(t_{m-1}, m-1\right),\left(t_{m-2}, m-2\right),\left(t_{m-3}, m-3\right), \ldots,\left(t_{z_{j}}, z_{j}\right),
$$

where $j \leq t_{m-1} \leq t_{m-2} \leq \ldots \leq t_{z_{j}}$.

(iv) The $z$-marks in a given column with number smaller than $m$ strictly increase from top to bottom.

(v) In $D_{\mu}^{t}$ only pure $v$-boxes or $\bar{v}$-boxes appear, and in a fixed column their marks strictly increase from top to bottom.

(vi) If a D-box $\mathfrak{a}$ appears in column $m$, then it has $z$-marks $i, i+1$, or a certain $z$-mark and $a$-mark $\alpha$, or a certain $z$-mark and a $\bar{v}$-mark. If $\mathfrak{b}$ is a D-box in column $m$ and the row of $\mathfrak{a}$ is above the row of $\mathfrak{b}$, then (each) $z$-mark of $\mathfrak{a}$ is smaller than (each) z-mark of $\mathfrak{b}$. 
This proposition describes the $z$-ribbons in $\stackrel{\circ}{D}_{\mu}^{b}$. When depicting a $z$ ribbon, instead of drawing its boxes $(i, m),(i+1, m-1)$, we shall draw the boxes $(i+1, m),(i+1, m-1)$ in $D_{\mu}^{b}$.

We want now to discuss the restrictions of $v$ - and $\bar{v}$-ribbons to $\stackrel{\circ}{D}_{\mu}^{b}$. For simplicity of exposition we shall often consider them in $D_{\mu}^{b}$ using "simplifications" indicated by the following pictures: in $D_{\mu}^{b}$,
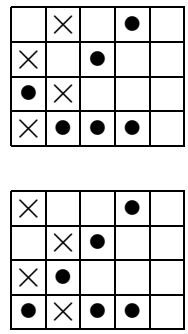

real ribbon
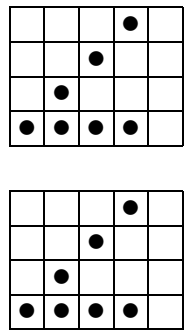

ribbon after "simplification"

Using this convention, the restrictions of $v$ - and $\bar{v}$-ribbons in $\stackrel{\circ}{D}_{\mu}^{b}$ are described as in [PR2, Proposition 4.7]. (The function $y(x)$ there is decreasing on pure $\bar{v}$-boxes and increasing on non-pure $\bar{v}$-boxes.)

For numerous examples of ribbons, see [Exx]. Schematic pictures of "typical" ribbons of different kinds are:

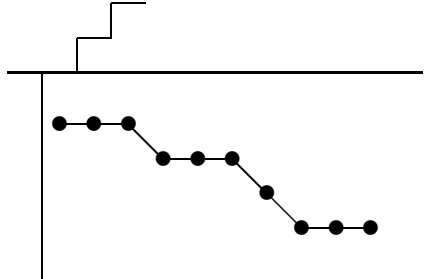

$z$-ribbon

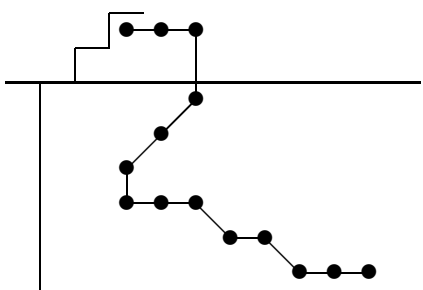

$v$-ribbon

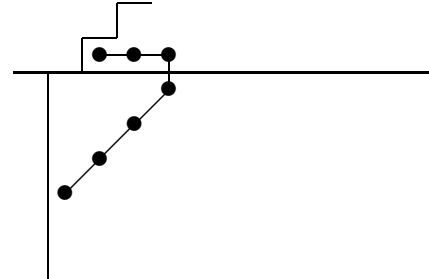

$\bar{v}$-ribbon

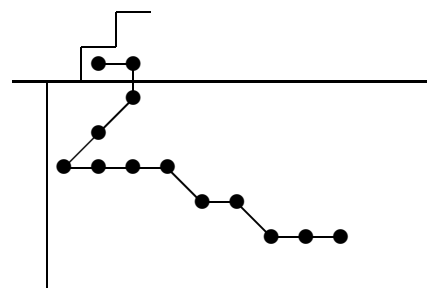

$\bar{v} / v$-ribbon

We have two basic operations of deforming $z$-ribbons.

- ("Push down") Suppose that $i$ is odd and the boxes

$$
(i, m),(i, m-2), \ldots,(i, j)
$$


form an entire $z$-ribbon. The operation transforms them to

$$
(i+2, m),(i+2, m-2), \ldots,(i+2, j) .
$$

Suppose that $i$ is even and the boxes

$$
(i, m-1),(i, m-2), \ldots,(i, j)
$$

form an entire $z$-ribbon. The operation transforms them to

$$
(i+2, m-1),(i+2, m-2), \ldots,(i+2, j) .
$$

(We assume that rows $i+1$ and $i+2$ contain no $D$-boxes before this operation.)

For example, the ribbons
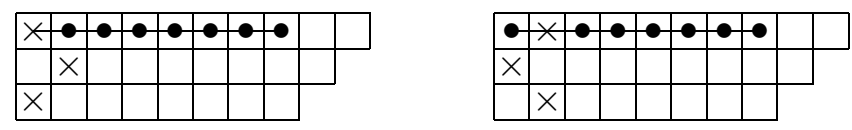

can be pushed down to
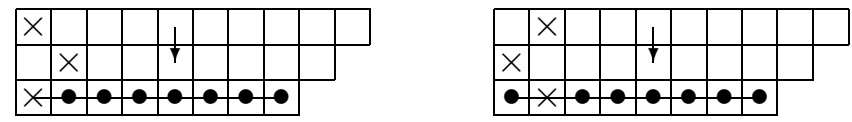

- ("Breaking a ribbon") Let $j \leq m-2$. The operation transforms a final segment

$$
(i, j),(i, j-1), \ldots,(i, h)
$$

of a $z$-ribbon to the $\sim D$-boxes:

$$
(i+1, j),(i+1, j-1), \ldots,(i+1, h),
$$

provided either $(i+1, j+1)$ is a $\sim D$-box, or it is $\times$ and $(i+1, j+2)$ is a $\sim D$-box. The box $(i, j)$ (before the operation) is called the breaking box.

For example, for $\mathfrak{a}$ a breaking box and $\mathfrak{b}$ a $\sim D$-box, or $\mathfrak{b}=\times$ and $\mathfrak{c}$ a $\sim D$-box:

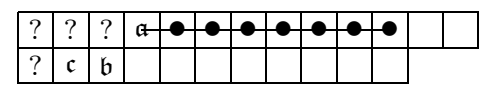

can be broken at $\mathfrak{a}$ and transformed to

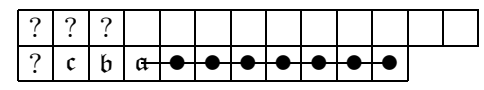

We shall take suitable compositions of these operations. 
Definition 3.8 ("Maximal deformation" of $D_{\lambda}^{b} \subset D_{\mu}^{b}$ ).

- Pick the lowest $z$-ribbon. Push it down as many times as possible. Then choose the leftmost breaking box on this ribbon (if it exists) and break the ribbon.

- Pick a $z$-ribbon and suppose that all lower $z$-ribbons in $D_{\lambda}^{b}$ have already been deformed. Push down this ribbon as many times as possible. Let $\mathfrak{a}$ be the leftmost breaking box on the ribbon. Break the ribbon at $\mathfrak{a}$ as many times as possible. Then choose the next leftmost breaking box $\mathfrak{b}$ and break the ribbon at $\mathfrak{b}$ as many times as possible etc.

In Section 7 we shall apply the maximal deformation to some (slightly) more general subsets of $D_{\mu}^{b}$ than Ferrers diagrams.

There is still another transformation of reduced decompositions that we shall need. Suppose that $\lambda$ is of type 2 and $D_{\lambda}^{b} \subset D_{\mu}^{b}$. Let $D \subset \stackrel{\circ}{D}_{\mu}$ be such that $r_{D} \in R\left(w_{\lambda}\right)$. For the purpose of the next definition, we shall call the maximal lowest segment of the $\bar{v} / v$-ribbon consisting entirely of $v_{\alpha}$-boxes the lowest $v_{\alpha}$-part of the $\bar{v} / v$-ribbon. We assume that

- the lowest $v_{\alpha}$-part of the $\bar{v} / v$-ribbon is a row in $D$, but not the first one in the bottom part;

- all $z$-ribbons above the lowest $v_{\alpha}$-part of the $\bar{v} / v$-ribbon are rows in $D$.

We now give the following definition.

DeFinition 3.9 (An "e-transformation").

- Exchange the lowest $v_{\alpha}$-part of the $\bar{v} / v$-ribbon with the next row of $D$ above it, increasing the former by one $D$-box.

- Remove the rightmost box from the $\alpha$ th row of $D^{t}$.

(We assume that all these operations are feasible in $D_{\mu}$; otherwise the etransformation of $D$ changes nothing.)

Here is a picture of an e-transformation with the (simplified) $\bar{v} / v$-ribbons depicted with " $\star$ ".
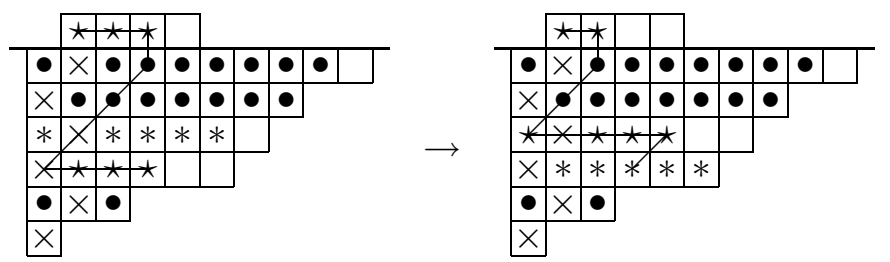

One readily checks that if $D^{\prime}$ is obtained from $D$ via an e-transformation, then $r_{D^{\prime}} \in R\left(w_{\lambda}\right)$.

We finish this section with the following simple consequence of Proposition 3.2 . 
LEMma 3.10. Assume that $r_{D} \in R\left(w_{\lambda}\right)$. The following configurations of boxes cannot appear in $\stackrel{\circ}{D}_{\mu}^{b}$ :

\begin{tabular}{|l|l|}
\hline $\mathfrak{c}$ & $\mathfrak{b}$ \\
\hline $\mathfrak{a}$ &
\end{tabular}

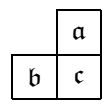

where $\mathfrak{a}$ is a D-box, $\mathfrak{b}$ is a D-box, and $\mathfrak{c}$ is a $z$-box.

Proof. Suppose, for instance, that $\mathfrak{a}$ is a pure $v$-box with mark $j$. The operator of $\mathfrak{a}$ moves $v_{j}$ forward and a certain $x$ goes backward:

$$
\left[\ldots, v_{j}, x, \ldots\right] \rightarrow\left[\ldots, x, v_{j}, \ldots\right],
$$

so $x$ cannot be equal to any $z$ by Proposition 3.2. But $\mathfrak{c}$ is a $z$-box with mark $i$, say, and its operator moves $x$ forward, so $x=z_{i}$. We get a contradiction.

4. Schubert classes and the characteristic map. We now pass to homogeneous spaces. We shall use the following notation:

- $P_{n}$-the maximal parabolic subgroup of $H=S O(2 m)$ containing $B$ and corresponding to the subset $\Sigma \backslash\left\{\varepsilon_{n}-\varepsilon_{n+1}\right\}$ of $\Sigma$ (here, as always in this paper, $1 \leq n<m$ ),

- $F=H / B$ - the flag manifold of complete isotropic flags,

- $G=H / P_{n}$-an isotropic orthogonal Grassmannian.

$G$ can be interpreted as the variety of $(n-1)$-spaces lying on a $(2 m-2)$ dimensional smooth quadric. According to Segre [Se] and [GH, p. 739], the dimension of the latter variety is $n(2 m-n)-n(n+1) / 2$ (this is in agreement with Remark 3.4). Also, the Euler characteristic of $G$ is $\# W^{(n)}=2^{n}\left(\begin{array}{c}m \\ n\end{array}\right)$. For the Betti numbers of $G$, see Proposition 4.1 below. Let

$$
c: S^{\bullet}(X(T) \otimes \mathbb{Q})=\mathbb{Q}\left[x_{1}, \ldots, x_{m}\right] \rightarrow H^{*}(F)
$$

be the Borel characteristic map [Bo]. (We always take the cohomology with rational coefficients; in fact the coefficients from $\mathbb{Z}[1 / 2]$ would suffice.) By $[\mathrm{BGG}]$ and $[\mathrm{D} 2], c$ induces a surjective map

$$
c_{G}: \mathbb{Q}[X]^{W_{n}} \rightarrow H^{*}(G)
$$

where $X=\left\{x_{1}, \ldots, x_{m}\right\}$. We check as in the proof of [PR2, Theorem 1.4] that the ideal $\operatorname{Ker}(c)=\left(e_{i}\left(x_{1}^{2}, \ldots, x_{m}^{2}\right), i<m, e_{m}(X)\right)$ generated by the $W$-invariants is $W_{n}$-invariant $\left(e_{i}(-)\right.$ denotes the $i$ th elementary symmetric polynomial). Thus we have

$$
H^{*}(G) \simeq \frac{\mathcal{S P}\left(x_{1}, \ldots, x_{n}\right) \otimes_{\mathbb{Q}} \mathcal{S P}\left(x_{n+1}^{2}, \ldots, x_{m}^{2}\right)[u]}{\left(e_{i}\left(x_{1}^{2}, \ldots, x_{m}^{2}\right), i<m, e_{m}(X)\right)},
$$

where $u=x_{n+1} \cdots x_{m}$ and $\mathcal{S P}(-)$ denotes the ring of symmetric polynomials (here with rational coefficients). 
Proposition 4.1. The Poincaré series $P(t)=\sum_{i} \operatorname{dim}_{\mathbb{Q}} H^{2 i}(G) t^{i}$ is equal to

$$
P(t)=\frac{\left(1-t^{2 \alpha}\right) \cdots\left(1-t^{2(m-1)}\right)\left(1-t^{m}\right)}{(1-t) \cdots\left(1-t^{n}\right)\left(1-t^{\alpha}\right)} .
$$

Proof. The elements $e_{i}\left(x_{1}^{2}, \ldots, x_{m}^{2}\right), i=1, \ldots, m-1$, and $e_{m}\left(x_{1}, \ldots, x_{m}\right)$ are algebraically independent. The Poincaré series of $\mathcal{S P}\left(x_{1}, \ldots, x_{n}\right)$ and $\mathcal{S P}\left(x_{n+1}^{2}, \ldots, x_{m}^{2}\right)[u]$ are equal to

$$
\frac{1}{(1-t) \cdots\left(1-t^{n}\right)} \quad \text { and } \quad \frac{1}{\left(1-t^{2}\right) \cdots\left(1-t^{2 \beta}\right)\left(1-t^{\alpha}\right)}
$$

respectively. Hence

$$
\begin{aligned}
P(t) & =\frac{\left(1-t^{2}\right)\left(1-t^{4}\right) \cdots\left(1-t^{2 \alpha}\right) \cdots\left(1-t^{2(m-1)}\right)\left(1-t^{m}\right)}{(1-t) \cdots\left(1-t^{n}\right)\left(1-t^{2}\right)\left(1-t^{4}\right) \cdots\left(1-t^{2 \beta}\right)\left(1-t^{\alpha}\right)} \\
& =\frac{\left(1-t^{2 \alpha}\right) \cdots\left(1-t^{2(m-1)}\right)\left(1-t^{m}\right)}{(1-t) \cdots\left(1-t^{n}\right)\left(1-t^{\alpha}\right)}
\end{aligned}
$$

(Cf. also $[\mathrm{AC}]$ and the references there.)

Using the above presentation of $H^{*}(G)$, and arguing as in the proof of [PR2, Theorem 1.4], we get the following

Corollary 4.2. The images via $c_{G}$ of the elements $e_{i}\left(x_{1}, \ldots, x_{n}\right)$, for $i=1, \ldots, n$, and of u generate multiplicatively the ring $H^{*}(G)$.

For a given $\mathbf{a}=\left(a_{m}, a_{m-1}, \ldots, a_{2}, a_{1}\right) \in\{-1,0,1\}^{m}$, we define the generating function

$$
E_{\mathbf{a}}=\prod_{i=1}^{m}\left(1+a_{i} x_{i}\right) .
$$

In particular, for $\mathbf{a}=(0, \ldots, 0,1, \ldots, 1)$ with $\alpha$ zeros and $n$ ones, the resulting generating function, denoted by $E$, is the generating function for the elementary symmetric polynomials in $x_{1}, \ldots, x_{n}$. We record some simple properties of $s$ - and $\partial$-operations applied to these generating functions (cf. [PR2] and [DP]).

Lemma 4.3. (a) We have $s_{i}\left(E_{\mathbf{a}}\right)=E_{\mathbf{a}^{\prime}}$, where

$$
\mathbf{a}^{\prime}= \begin{cases}\left(a_{m}, \ldots, a_{i+2}, a_{i}, a_{i+1}, a_{i-1}, \ldots, a_{1}\right), & i<m, \\ \left(-a_{m-1},-a_{m}, a_{m-2}, \ldots, a_{1}\right), & i=m .\end{cases}
$$

(b) For $i=1, \ldots, m-1$,

$$
\partial_{i}\left(E_{\mathbf{a}}\right)=d \cdot E_{\mathbf{a}^{\prime}} \quad \text { if } a_{i}=a_{i+1}+d \quad(d=-2,-1,0,1,2),
$$

where $\mathbf{a}^{\prime}=\left(a_{m}, \ldots, 0,0, \ldots, a_{1}\right)$ is the sequence $\mathbf{a}$ with $a_{i+1}, a_{i}$ replaced by zeros.

(c) $\partial_{m}\left(E_{\mathbf{a}}\right)=\left(a_{m}+a_{m-1}\right) \cdot E_{\left(0,0, a_{m-2}, \ldots, a_{1}\right)}$. 
In particular, if $\Delta$ is a composition of some $s$ - and $\partial$-operations, then for every $\mathbf{a}, \Delta\left(E_{\mathbf{a}}\right)=($ scalar $) \cdot E_{\mathbf{a}^{\prime}}$, where $\mathbf{a}^{\prime}$ is uniquely determined if this scalar is not zero.

Denoting by $B^{-}$the Borel subgroup opposite to $B$, let $X_{w}=\left[\overline{B^{-} w B / B}\right]$ be the Schubert class in $H^{2 l(w)}(F)$ corresponding to $w \in W$. Then, for a homogeneous polynomial $f \in \mathbb{Q}[X]$, we have (cf. [BGG], [D1], [D2])

$$
c(f)=\sum_{l(w)=\operatorname{deg} f} \partial_{w}(f) X_{w} .
$$

If $\lambda$ is a shape and $r=l\left(w_{\lambda}\right)$, then $X_{w_{\lambda}} \in H^{2 r}(G) \subset H^{2 r}(F)$, and we shall denote this element in $H^{2 r}(G)$ by $\sigma(\lambda)$.

EXAMPLE 4.4. When $n=1, G$ is a quadric hypersurface in $\mathbb{P}^{2 m-1}$, and the poset of Schubert classes has a particularly simple structure. Namely, there are $2 m$ Schubert classes, one in each dimension, except for the middle dimension $m-1$ where there are two classes (those of codimension $<m-1$ are of type 1 , those of codimension $>m-1$ are of type 2 , and the two in dimension $m-1$ represent both types). The multiplication by the class of a hypersurface is well known (in $[\mathrm{H}]$ it is interpreted as a particular case of Chevalley's formula [C]). Take for simplicity $m=3$. Then $\operatorname{dim} G=4$ and one has 6 Schubert classes: $1=\sigma(2,1 / / \emptyset), h=\sigma(3,1 / / \emptyset)$ (the class of a hyperplane), $a=\sigma(3,1 / / 2,1), b=\sigma(3,2 / / \emptyset), l=\sigma(3,2 / / 2,1)$ (the class of a line), and $*=\sigma(3,2 / / 3,1)$ (the class of a point). We have: $1 \cdot h=h$, $h^{2}=a+b, a \cdot h=l, b \cdot h=l$, and $l \cdot h=*$.

Note that for $p=1, \ldots, n$,

$$
\begin{aligned}
X_{s_{n-p+1} \cdots s_{n-1} \cdot s_{n}} & =X_{[1, \ldots, n-p, n-p+2, \ldots, n+1 ; \emptyset ; n-p+1, n+2, \ldots, m]} \\
& =\sigma(\alpha+p, \alpha-1, \alpha-2, \ldots, 2,1 / / \emptyset)=: \sigma_{p} .
\end{aligned}
$$

By a direct calculation we get:

Lemma 4.5. For $p=1, \ldots, n$, one has $c\left(e_{p}\left(x_{1}, \ldots, x_{n}\right)\right)=\sigma_{p}$.

Given a shape $\lambda$, we pick a homogeneous $f_{\lambda} \in \mathbb{Q}[X]$ such that $c\left(f_{\lambda}\right)=$ $\sigma(\lambda)$. Then, for $w \in W$ with $l(w)=l\left(w_{\lambda}\right)$, one has $\partial_{w}\left(f_{\lambda}\right) \neq 0$ iff $w=w_{\lambda}$ and $\partial_{w_{\lambda}}\left(f_{\lambda}\right)=1$. We want to find the coefficients $d_{\mu}$ in the expansion

$$
\sigma(\lambda) \cdot \sigma_{p}=c\left(f_{\lambda} \cdot e_{p}\left(x_{1}, \ldots, x_{n}\right)\right)=\sum d_{\mu} \sigma(\mu) .
$$

For every $f, g \in \mathbb{Z}[X]$ and any $i$, we have a Leibniz-type formula

$$
\partial_{i}(f \cdot g)=f \cdot\left(\partial_{i} g\right)+\left(\partial_{i} f\right) \cdot\left(s_{i} g\right) .
$$

Iterating (2) and invoking the operators $\partial_{\mu}^{D}$, one gets:

Proposition 4.6 (cf. [PR1], [PR2], [P1], [DP]). One has

$$
d_{\mu}=\sum \partial_{\mu}^{D}\left(e_{p}\left(x_{1}, \ldots, x_{n}\right)\right)
$$

where the sum is over all subsets $D \subset \stackrel{\circ}{D}_{\mu}$ such that $r_{D} \in R\left(w_{\lambda}\right)$. 
(A similar formula appeared in the paper [KK] by Kostant and Kumar.) We shall show that at most one non-zero summand can appear in the sum from the proposition, and compute $d_{\mu}$ in the case when such a unique nonzero term appears.

5. When does $\partial_{\mu}^{D}(E)$ vanish? In this section, we describe configurations of $D$ - and $\sim D$-boxes in $D_{\mu}$ for which $\partial_{\mu}^{D}(E)=0$. We then say that a configuration causes vanishing.

Recalling that $E=E_{\mathbf{a}}$ where $a_{m}=\ldots=a_{n+1}=0$, one easily gets:

Lemma 5.1. The following configuration of two $\sim D$-boxes in $D_{\mu}^{t}$ causes vanishing:

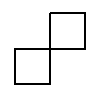

Arguing as in the proof of [PR2, Lemma 5.5], one obtains:

Lemma 5.2. If $\partial_{\mu}^{D}(E) \neq 0$, then the top segment of a $v$-ribbon (resp. the $\bar{v}$-ribbon) is of the form

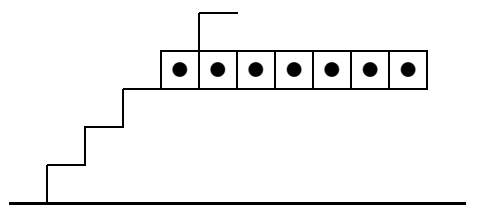

Corollary 5.3. If $r_{D} \in R\left(w_{\lambda}\right)$ and $\partial_{\mu}^{D}(E) \neq 0$, then

(i) the lengths of the consecutive rows in $D^{t}$ are decreasing;

(ii) $D_{\mu}^{t} \backslash D^{t}$ is a horizontal strip with pairwise separated rows.

Proof. (i) Otherwise there exists an $s$-operation which transposes two $v$ 's-but this contradicts Proposition 3.2.

(ii) This follows from Lemmas 5.1 and 5.2.

Lemma 5.4. Let $\partial_{\mu}^{D}(E) \neq 0$ and $\Delta$ be the "part" of $\partial_{\mu}^{D}$ formed by the composition of the $s$ - and $\partial$-operators of the boxes from $\stackrel{\circ}{D}_{\mu}$. Then $\Delta(E)=$ $1 \cdot E_{\mathbf{a}}$, where the sequence $\mathbf{a}=\left(a_{m}, \ldots, a_{1}\right)$ is defined as follows:

$a_{h}=\left\{\begin{array}{l}0 \quad \text { if } h \text { is the column number of the rightmost box of a row of } D^{t}, \\ 0 \quad \text { if } h \text { is the column number of a box in } D_{\mu}^{t} \backslash D^{t}, \\ 1 \quad \text { in the remaining cases. }\end{array}\right.$

It is convenient to introduce now the following two operators. For a given box $\mathfrak{a} \in \stackrel{\circ}{D}_{\mu}$, let $\dot{\Delta}_{\mathfrak{a}}$ be the composition of the operators of the boxes preceding $\mathfrak{a}$ in $\stackrel{\circ}{D}_{\mu}$ when reading $\stackrel{\circ}{D}_{\mu}$ row by row from left to right and from top to bottom. By $\Delta_{\mathfrak{a}}$ we denote the composition of $\Delta_{\mathfrak{a}}$ and the operator of $\mathfrak{a}$ in $\stackrel{\circ}{D}_{\mu}$. 
It is clear that if $\partial_{\mu}^{D}(E)=0$, then there exists a $\sim D$-box $\mathfrak{a}$ such that

$$
\dot{\Delta}_{\mathfrak{a}}(E) \neq 0 \quad \text { and } \quad \Delta_{\mathfrak{a}}(E)=0 .
$$

Such a box will be called bad. Obviously, $\partial_{\mu}^{D}(E) \neq 0$ iff there are no bad boxes in $D_{\mu} \backslash D$. Corollary 5.3 gives necessary (and sufficient) conditions for the absence of bad boxes in $D_{\mu}^{t}$.

Consider the following schematic pictures of two configurations of boxes:
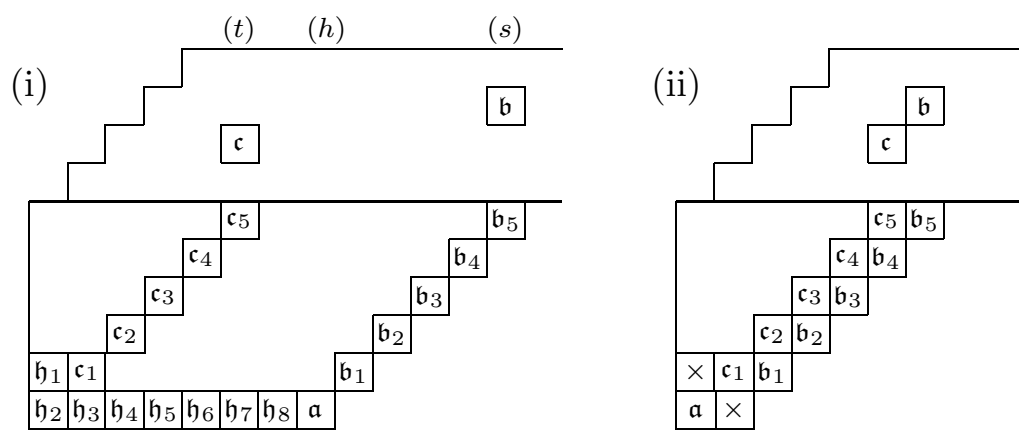

Note that the bottom parts of these configurations are completely determined by $\mathfrak{a}$. In both pictures, the box $\mathfrak{b}$ is the lowest box of $D_{\mu}^{t}$ in the column of the box $\mathfrak{b}_{5}$; similarly the box $\mathfrak{c}$ is the lowest box of $D_{\mu}^{t}$ in the column of the box $\mathfrak{c}_{5}$.

In the following proposition, we tacitly assume that $\stackrel{\triangle}{\mathfrak{a}}_{(}(E) \neq 0$.

Proposition 5.5. (i) Suppose that the column number of $\mathfrak{a}$ is $h<m$. Then $\mathfrak{a}$ is bad if and only if at least one of the following conditions holds:

- $\mathfrak{b}$ and $\mathfrak{c}$ are $\sim D$-boxes or the rightmost $D$-boxes in their rows;

- $\mathfrak{b}$ is a D-box or the rightmost D-box in its row, and some $\mathfrak{c}_{i}$ is a $\sim D$-box or some $\mathfrak{h}_{j}$ is a $\sim D$-box;

- $\mathfrak{c}$ is a D-box or the rightmost D-box in its row, and some $\mathfrak{b}_{i}$ is a $\sim D$-box;

- some $\mathfrak{c}_{i}$ and $\mathfrak{b}_{j}$, or some $\mathfrak{h}_{i}$ and $\mathfrak{b}_{j}$, are $\sim D$-boxes.

(ii) Suppose that the column number of $\mathfrak{a}$ is $m$. Then $\mathfrak{a}$ is bad if and only if at least one of the following conditions holds:

- $\mathfrak{b}$ and $\mathfrak{c}$ are $\sim D$-boxes or the rightmost boxes in their rows;

- $\mathfrak{b}$ is a D-box or the rightmost box in its row, and some $\mathfrak{c}_{j}$ is a $\sim D$-box;

- $\mathfrak{c}$ is a D-box or the rightmost box in its row, and some $\mathfrak{b}_{i}$ is a $\sim D$-box.

Proof. (i) Let $E_{\mathbf{a}}$ be the function obtained from $E$ after applying the operators of the boxes of $D_{\mu}^{t}$ and let $\Delta_{\mathfrak{a}}^{b}$ be the composition of all operators of the boxes preceding $\mathfrak{a}$ in $D_{\mu}^{b}$ when reading $D_{\mu}^{b}$ row by row from left to 
right and from top to bottom. We want to calculate the components $a_{h}^{\prime}, a_{h+1}^{\prime}$ in the sequence $\mathbf{a}^{\prime}$ defined by $\Delta_{\mathfrak{a}}^{b}\left(E_{\mathbf{a}}\right)=($ scalar $) \cdot E_{\mathbf{a}^{\prime}}$. Clearly, $\mathfrak{a}$ is bad iff $a_{h}^{\prime}=a_{h+1}^{\prime}$ (in fact, it will be clear from the discussion below that $\mathfrak{a}$ is bad iff $\left.a_{h}^{\prime}=a_{h+1}^{\prime}=0\right)$.

Assume that $\mathbf{a}=(\ldots, c, \ldots, b, \ldots)$, where $b=a_{s}$ and $c=a_{t}(s<t$ are the column numbers of $\mathfrak{b}$ and $\mathfrak{c}$, respectively). We know that $b$ and $c$ are 1 or 0 (Lemma 5.4). Note that in $\Delta_{\mathfrak{a}}^{b}$, only the operators of the $\mathfrak{b}$ 's influence $b$, and only the operators of the $\mathfrak{c}$ 's and $\mathfrak{h}$ 's influence $c$. One has $b=0$ (resp. $c=0$ ) iff $\mathfrak{b}$ (resp. $\mathfrak{c}$ ) is a $\sim D$-box or the rightmost box in a row in $D_{\mu}^{t}$.

Clearly, $a_{h+1}^{\prime}=a_{h}^{\prime}=0$ iff one of the following possibilities hold:

(1) $b=c=0$;

(2) $c=0$ and some $\mathfrak{b}_{i}$ is a $\sim D$-box;

(3) $b=0$ and some $\mathfrak{c}_{i}$ is a $\sim D$-box or some $\mathfrak{h}_{i}$ is a $\sim D$-box;

(4) some $\mathfrak{b}_{i}$ is a $\sim D$-box and some $\mathfrak{h}_{j}$ is a $\sim D$-box, or some $\mathfrak{b}_{i}$ is a $\sim D$-box and some $\mathfrak{c}_{j}$ is a $\sim D$-box.

Observe that the operation associated with $\mathfrak{h}_{1}$ or $\mathfrak{h}_{2}$ changes the sign of $c$ so $a_{h}^{\prime}=-1$ or 0 and $a_{h+1}^{\prime}=0$ or 1 (see Lemma 5.4). Thus, in fact, the only possibility for the equality $a_{h+1}^{\prime}=a_{h}^{\prime}$ to hold is $a_{h+1}^{\prime}=a_{h}^{\prime}=0$.

This proves assertion (i). The proof of (ii) is analogous and we omit it.

This result will be very often used in what follows. It would increase the length of the paper enormously to specify, each time, the precise conditions which cause vanishing. Instead, we shall use some phrases in quotation marks to describe the reasons roughly. Their meaning will be explained now.

Assume that $\mathfrak{a}$ lies in column $h, h<m$. Let $\mathbf{b}$ and $\mathbf{c}$ be the sequences associated with $\mathfrak{b}$ and $\mathfrak{c}$. We see that the zero from the $s$ th place in $\mathbf{b}$ is transported to the $(h+1)$ st place in $\mathfrak{a}$, and the zero from the $t$ th place in $\mathbf{c}$ is transported to the $h$ th place in a. Then the operator $\partial_{h}$ of $\mathfrak{a}$ kills the generating function. We shall say: "A zero of $\mathfrak{b}$ comes to $\mathfrak{a}$ from the left, a zero of $\mathfrak{c}$ comes to $\mathfrak{a}$ from above", and $\mathfrak{a}$ causes vanishing.

Similarly, for $\mathfrak{a}$ in column $m$, we shall say: "A zero comes from above to $\mathfrak{a}$ and another zero comes from above to its right neighbor", and $\mathfrak{a}$ causes vanishing.

Let now $\mathfrak{d}$ be the rightmost box of a $v$-ribbon (resp. the $\bar{v} / v$-ribbon), in column $q$, say, in some bottom row. Suppose that $\Delta_{\mathfrak{o}}(E)=c \cdot E_{\mathbf{b}}$, where $\mathbf{b}=\left(b_{1}, \ldots, b_{m}\right)$ and $c$ is a non-zero scalar. Then $b_{q}=0$. We shall say: "A $v$-ribbon (resp. the $\bar{v} / v$-ribbon) brings a zero to $\mathfrak{d}$ ". In fact, "a zero often comes from the left to a box $\mathfrak{a}$ " because "the $\bar{v} / v$-ribbon brings it to the left neighbor of $\mathfrak{a}$ ", or simply: "the $\bar{v} / v$-ribbon brings a zero to $\mathfrak{a}$ from the left".

From such phrases in quotation marks, the reader will find the precise conditions causing vanishing with no difficulty. 
It is clear from Section 4 that if $\partial_{\mu}^{D}(E)=d \cdot E_{\mathbf{a}}$, then the scalar $d$ is a power of 2. Suppose that $\Delta_{\mathfrak{a}}(E)=E_{\mathbf{a}}$ for some $\sim D$-box $\mathfrak{a}$. We shall say that $\mathfrak{a}$ is essential if $\partial_{h}\left(E_{\mathbf{a}}\right)=2 \cdot E_{\mathbf{a}^{\prime}}$, where $\partial_{h}$ is the operator of $\mathfrak{a}$. As a consequence of the proof of Proposition 5.5, we have the following corollary (we use the notation from the pictures before the proposition).

Corollary 5.6. A box $\mathfrak{a}$ is essential if and only if:

(i) $\mathfrak{b}$ and $\mathfrak{c}$ are $D$-boxes but not the rightmost boxes in their rows; all boxes $\mathfrak{c}_{i}, \mathfrak{h}_{j}$ are $D$-boxes or $\times$-boxes; and all boxes $\mathfrak{b}_{i}$ are $D$-boxes; or

(ii) $\mathfrak{b}$ and $\mathfrak{c}$ are $D$-boxes but not the rightmost boxes in their rows; and all boxes $\mathfrak{b}_{i}$ and $\mathfrak{c}_{i}$ are $D$-boxes.

6. Some terminology and the main result. By a skew diagram we shall understand a difference of the diagrams of two strict partitions. We use the following conventions for "strips":

A 1-strip is an (ordinary) horizontal strip (that is, a skew diagram with at most one box in each column).

A 1/2-strip is a horizontal strip with pairwise separated rows.

A degenerate strip is a strip which is horizontal and vertical.

A 3/2-strip is a (possibly disconnected) skew diagram with at most two boxes in each column, but with exactly 1 box in its leftmost column. Every $3 / 2$-strip has a decomposition into connected components. The set of nonhighest boxes in columns of a component forms a set called the excrescence of the component. Observe that no box from the excrescence lies in the leftmost column of the component. (A 3/2-strip is an "almost horizontal strip" in the terminology of [PR2]. We shall mostly use connected 3/2-strips.)

Note that 1 -strips, $1 / 2$-strips and degenerate strips are $3 / 2$-strips.

A 2-strip is a connected skew diagram having at most 2 boxes in one column, but exactly 2 boxes in the leftmost column. Consider the largest family of initial columns, each having 2 boxes. The boxes from these columns form the proper 2 -strip of the 2 -strip. The remaining boxes form a $3 / 2$-strip which will be called the $3 / 2$-strip of the 2 -strip.

In the following picture, we exhibit a typical 2-strip:

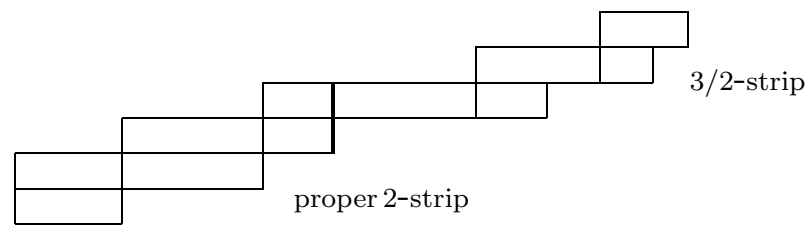

A 2-strip whose proper 2-strip consists of a single column ${ }_{\times}^{\circ}$ will be called an almost 3/2-strip. 
Suppose that $1 \leq n<m$ are fixed, and two shapes $\lambda=\left(\lambda^{t} / / \lambda^{b}\right)$ and $\mu=$ $\left(\mu^{t} / / \mu^{b}\right)$ are given (cf. Definition 2.1). We adopt verbatim the terminology from [PR2, Section 2] for such notions and expressions as: $\lambda$-part of a row, $\mu$ part of a row, exceptional row, $(\mu-\lambda)$-box (all these refer to objects from the top part); as well as: component, extremal component, lying/appearing over, and ending over. Each time the word "over" will be used in this "shifted" sense from [PR2], we shall write it in boldface.

Note: The boxes of a component of $D_{\mu}^{b} \backslash D_{\lambda}^{b}$ equipped with "×" are treated as "true" boxes of the component.

We shall write $\lambda_{\alpha}^{t}$ (resp. $\mu_{\alpha}^{t}$ ) for the $\lambda$-part (resp. $\mu$-part) of the $\alpha$ th (top) row.

Suppose that $\lambda$ (resp. $\mu$ ) is of type 2. The $\lambda_{\alpha}^{t}$ th (resp. $\mu_{\alpha}^{t}$ th) row of $D_{\lambda}^{b}$ (resp. $D_{\mu}^{b}$ ) will be called the special $\lambda$-row (resp. the special $\mu$-row). Note that $\lambda_{\alpha}^{t}$ (resp. $\mu_{\alpha}^{t}$ ) ends precisely over the leftmost box of the special $\lambda$-row (resp. the special $\mu$-row). For example, if $\lambda=(15,6 / / 16,15,13,10,8,7,5,2)$, then the 6 th bottom row is the special $\lambda$-row:

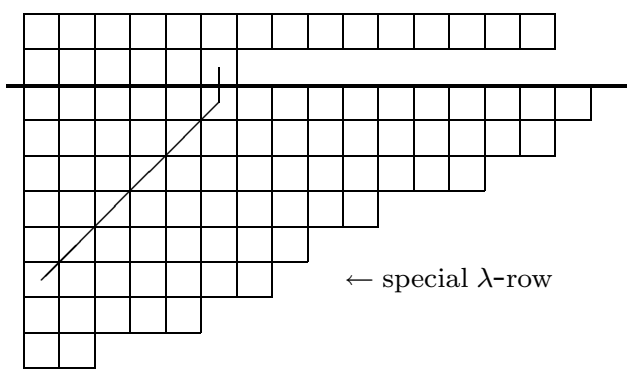

If $w_{\lambda}$ is of type 2 , then the component meeting the special $\lambda$-row will be called special. If $w_{\lambda}$ is of type 1 and $w_{\mu}$ is of type 2 , then the component meeting the special $\mu$-row will be called special.

DEFINITION 6.1. $\mu$ is compatible with $\lambda$ if the following conditions hold:

(i) $D_{\mu}^{b} \supset D_{\lambda}^{b}$ and every non-special, non-extremal component of $D_{\mu}^{b} \backslash D_{\lambda}^{b}$ is a $3 / 2$-strip. Moreover, $D_{\mu}^{t} \backslash D_{\lambda}^{t}$ is a $1 / 2$-strip.

(ii) The $\lambda$-part of at most one row from the top part ends over a component. Such a pair will be called related, i.e. we shall say that the row is related to the component (or simply "related"), and the component is related to the row (or simply "related").

(iii) Each exceptional row is related to a component over which its $\mu$-part ends.

(iv) If a $(\mu-\lambda)$-box lies over a component which is a $3 / 2$-strip, then this component is not related and this box lies over the leftmost box of the component. If a $(\mu-\lambda)$-box lies over the component which is a 2-strip, then this box lies over the highest leftmost box of this 2-strip. 
(v) An excrescence can appear only in a related component, under the $\lambda$-part of the related row; no box from the $\mu$-part of the related row lies over the excrescence.

Moreover, there are some conditions (C1.1), (C2.1), (C1.2), and (C2.2) for the special and extremal components to be satisfied. They will be given in subsections 7.1-7.4 below, according to types of $\lambda$ and $\mu$.

For compatible $\mu$ and $\lambda$, apart from the case when they are both of type 2 , we define $e(\lambda, \mu)$ to be the number of non-related components above the special one. If $\mu$ and $\lambda$ are of type 2 , the number $e(\lambda, \mu)$ is described in Proposition 7.40. Moreover, in all instances we require that no $(\mu-\lambda)$-boxes lie over a component contributing to $e(\lambda, \mu)$.

Theorem 6.2. For every shape $\lambda$ and $p=1, \ldots, n$,

$$
\sigma(\lambda) \cdot \sigma_{p}=\sum 2^{e(\lambda, \mu)} \sigma(\mu)
$$

where the sum is over all $\mu$ which are compatible with $\lambda$ and $l\left(w_{\mu}\right)=l\left(w_{\lambda}\right)+p$.

7. Proof of the theorem. In this section, we assume tacitly that $D \subset \stackrel{\circ}{D}_{\mu}$ is such that $r_{D} \in R\left(w_{\lambda}\right)$, and freely use the notions associated with such a $D$ in the earlier sections. In particular, whenever we speak about an $s$-operation we mean a simple reflection from the reduced decomposition $r_{D}$ of $w_{\lambda}$ encoded by $D$; all $z$ 's and $v$ 's refer to the corresponding symbols for $w_{\lambda}$. Moreover, we also assume that $\partial_{\mu}^{D}(E) \neq 0$. We shall call this condition "non-vanishing". In case some result holds true without the latter assumption, we shall mention it.

7.1. Case 1: $\lambda$ and $\mu$ are of type 1

Lemma 7.1. No s-operation supplies $v_{\alpha}$ with a bar.

Proof. Suppose that $v_{\alpha}$ receives a bar. Then it must lose it (there is no $v_{i}$ with a bar in $w_{\lambda}$ ). Consider the $\bar{v} / v$-ribbon whose relevant part is depicted below:

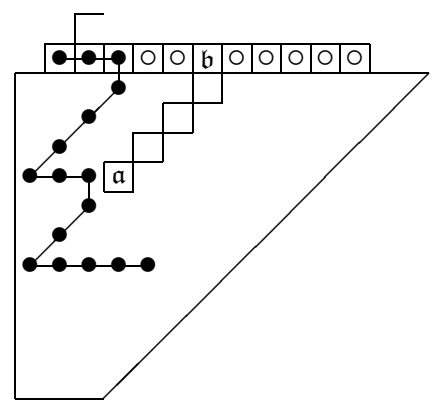

By the separation property, $\mathfrak{a}$ is a $\sim D$-box. The box $\mathfrak{b}$ lying over $\mathfrak{a}$ belongs to $\stackrel{\circ}{D}_{\mu}$ (because $\mu$ is of type 1 ) and it is a $\sim D$-box by Lemma 5.2 . We now 
use Proposition 5.5 in the following way $\left({ }^{3}\right)$. "The $\bar{v} / v$-ribbon brings a zero which comes from the left to $\mathfrak{a}$, another zero from $\mathfrak{b}$ comes to $\mathfrak{a}$ from above", and $\mathfrak{a}$ causes vanishing - a contradiction.

Proposition 7.2. The set of $z$-boxes is the maximal deformation of $D_{\lambda}^{b}$ in $D_{\mu}^{b}$.

Applying to the set of $z$-boxes the operations inverse to breaking rows and pushing rows down, we do not leave $D_{\mu}^{b}$ and get $D_{\lambda}^{b}$. Hence $D_{\lambda}^{b} \subset D_{\mu}^{b}$. (This argument does not require non-vanishing and applies also to Case 3.) Then, with the help of Lemma 3.10, the proof of the proposition is the same as the one of [DP, Proposition 3.10] (with " $n$ " there replaced by " $m$ ").

LEMMA 7.3. One has $\stackrel{i}{(}\left(\mu^{b}\right)-\stackrel{l}{l}\left(\lambda^{b}\right) \leq 1$.

Proof. Suppose that $i\left(\mu^{b}\right) \geq i\left(\lambda^{b}\right)+2$. Pick the highest, say $i$ th, row which contains a $\sim D$-box $\mathfrak{b}$ in column $m$ or $m-1$ :
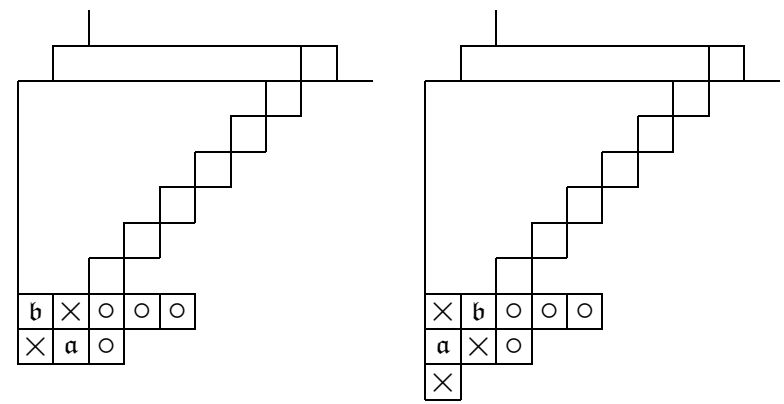

This is the highest row from which some ribbon has been pushed down in the maximal deformation, or, if there was no pushing down, it is row $l\left(\lambda^{b}\right)+1$. Since $\stackrel{i}{l}\left(\mu^{b}\right) \geq \stackrel{i}{l}\left(\lambda^{b}\right)+2$, and by a property of the maximal deformation, we see that row $i+1$ also contains a $\sim D$-box $\mathfrak{a}$ in column $m$ or $m-1$ respectively.

After breaking some higher ribbons we get the following configuration of $\sim D$-boxes:
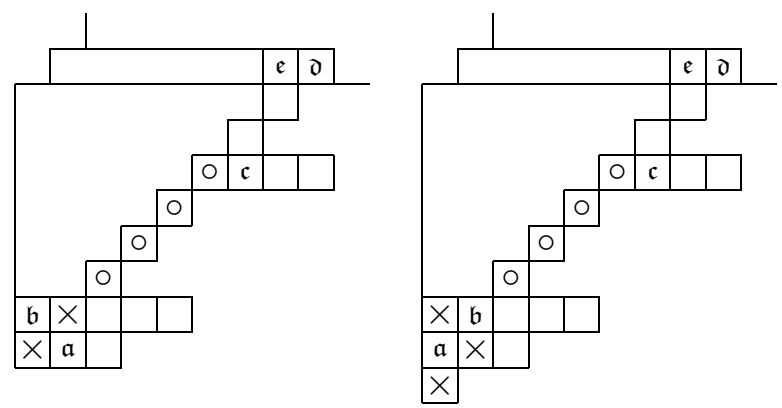

We shall use Proposition 5.5 several times.

$\left({ }^{3}\right)$ For the meaning of the phrases in quotation marks, see the discussion after Proposition 5.5. 
Consider first the picture on the left. If $\mathfrak{c}$ is a $\sim D$-box then "a zero comes from $\mathfrak{c}$ to $\mathfrak{a}$ from above, another zero comes from $\mathfrak{b}$ to $\mathfrak{a}$ from the left", and the $\sim D$-box $\mathfrak{a}$ causes vanishing - a contradiction. If $\mathfrak{c}$ is a $D$-box then it must be a $v$-box, and then $\mathfrak{e}$ is also a $v$-box, belonging to the same $v$-ribbon as $\mathfrak{c}$. By the separation property, $\mathfrak{d}$ is then a $\sim D$-box. We see that "a zero comes from $\mathfrak{d}$ to $\mathfrak{a}$ from above, another zero comes from $\mathfrak{b}$ to $\mathfrak{a}$ from the left", and $\mathfrak{a}$ causes vanishing - a contradiction.

Consider now the picture on the right. If $\mathfrak{c}$ is a $\sim D$-box then "a zero comes from $\mathfrak{c}$ to the right neighbor of $\mathfrak{a}$ from above, another zero comes from $\mathfrak{b}$ to $\mathfrak{a}$ from above", and $\mathfrak{a}$ causes vanishing - a contradiction. If $\mathfrak{c}$ is a $D$-box then it must be a $v$-box, and then $\mathfrak{e}$ is also a $v$-box, belonging to the same $v$-ribbon as $\mathfrak{c}$. By the separation property, $\mathfrak{d}$ is then a $\sim D$-box. We see that "a zero comes from $\mathfrak{d}$ to the right neighbor of $\mathfrak{a}$ from above, another zero comes from $\mathfrak{b}$ to $\mathfrak{a}$ from above", and $\mathfrak{a}$ causes vanishing. We get a contradiction again.

Since the push down requires the inequality $\stackrel{i}{l}\left(\mu^{b}\right)-\stackrel{\circ}{l}\left(\lambda^{b}\right) \geq 2$, we infer:

COROLlary 7.4. There is no push down operation in the maximal deformation. In particular, the breaking boxes can lie neither in column $m$ nor $m-1$.

As a consequence, the same proof as the one of [PR2, Proposition 6.2] gives us the following:

Proposition 7.5. The z-boxes with the same mark can appear in at most two successive rows.

In turn, the same arguments as those establishing [PR2, Proposition 6.7] show the following:

Proposition 7.6. (i) The non-extremal components of $D_{\mu}^{b} \backslash D_{\lambda}^{b}$ form a 3/2-strip.

(ii) (C1.1): The extremal component of $D_{\mu}^{b} \backslash D_{\lambda}^{b}$ is an almost 3/2-strip.

Here is a typical extremal component, depicted with "o", before and after the maximal deformation:
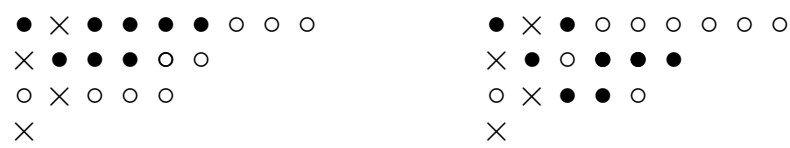

It follows from Proposition 7.6 that a typical deformed non-extremal component looks like: 


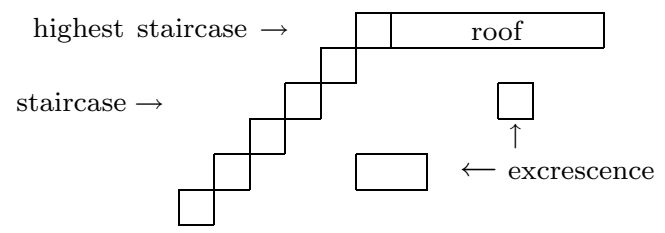

(cf. [PR2, end of Section 3]).

We have proved that if $\partial_{\mu}^{D}(E) \neq 0$, then the positions of $z$-boxes are uniquely determined. Now, we must determine the positions of pure $v$-boxes. We shall show that this non-vanishing condition can be satisfied for at most one $D$ (that is, the positions of $v$-boxes are also uniquely determined).

By arguing in (almost) the same way as in [PR2, Section 6], with the help of Proposition 5.5, we get the following facts:

(a) An excrescence can appear only under the roof of a deformed component and there are no two boxes of the excrescence lying one over the other. Moreover, the segment of a row between the staircase box and the excrescence must contain a $z$-box. (Cf. [PR2, Lemma 6.8].)

(b) No pure $v$-box can appear in an excrescence. (Cf. [PR2, Proposition 7.3].)

(c) A set of pure $v$-boxes can appear only in the roof of a deformed component and it forms a segment starting from the leftmost box of the roof. No two pure $v$-boxes with different marks can appear in the same roof. (Cf. [PR2, Proposition 7.3].)

(d) No two different roofs can contain pure $v$-boxes with the same mark. (Cf. [PR2, Proposition 7.4].)

(e) The marks of segments of pure $v$-boxes in the roofs of consecutive deformed components increase from top to bottom. (Cf. [PR2, Proposition 7.4].)

Proposition 7.7 (cf. [PR2, Theorem 8.1]). There exists at most one $D \subset D_{\mu}$ such that $r_{D} \in R\left(w_{\lambda}\right)$ and $\partial_{\mu}^{D}(E) \neq 0$.

The set $D$ from the proposition may be constructed in the following way (cf. $[\operatorname{Exx}, 4])$.

RECIPE 7.8. Let $\lambda, \mu$ be two shapes satisfying the conditions:

(1) $D_{\mu}^{b} \supset D_{\lambda}^{b}, D_{\mu}^{b} \backslash D_{\lambda}^{b}$ is a $3 / 2$-strip and $\stackrel{l}{l}\left(\mu^{b}\right)-i\left(\lambda^{b}\right) \leq 1$.

(2) The $\lambda$-part of at most one row from the top part ends over a component.

Then proceed as in (1) and (2) of [PR2, Recipe 8.4]. (This also applies to the extremal component.) 
LEMma 7.9 (cf. [PR2, Lemma 8.5]). $D_{\mu}^{t} \backslash D_{\lambda}^{t}$ is a 1/2-strip, and no $(\mu-\lambda)$-box lies over the staircase of a related component.

Proposition 7.10. There exists (a unique) $D \subset D_{\mu}$ such that $r_{D} \in$ $R\left(w_{\lambda}\right)$ and $\partial_{\mu}^{D}(E) \neq 0$ if and only if $\mu$ is compatible with $\lambda$.

Proof. Suppose first that there exists $D$ such that $\partial_{\mu}^{D}(E) \neq 0$. We shall show that $\mu$ is compatible with $\lambda$. Note that conditions (i)-(v) of Definition 6.1 hold true: (i) because of Lemma 7.9 and Proposition 7.6; (ii) by Recipe 7.8(2); (iii) by Recipe 7.8 and $D \subset D_{\mu}$; (iv) by Recipe 7.8 and Proposition 5.5; finally (v) by Proposition 5.5.

Assume, conversely, that conditions (i)-(v) of Definition 6.1 and (C1.1) hold. We shall prove that the set of bad boxes is empty. Any deformed component looks as in the pictures after Proposition 7.6. Consider a nonextremal component. It follows from Proposition 5.5 that no box from the staircase can be bad. Suppose that a component is not related. This means that no $\lambda$-row ends over a component and if a $\sim D$-box lies over a component, then this box lies over the highest staircase. Thus no box from the roof can be bad. If a component is related, then no box in the roof can be bad (use (iv) and Proposition 5.5), and no box from the excrescence can be bad (use (v) and Proposition 5.5). A reasoning for the extremal component is similar.

For compatible $\mu$ and $\lambda$, let $D^{\lambda, \mu}$ denote the unique $D$ from the proposition. Clearly, $d_{\mu}$ is the number defined by $\partial_{\mu}^{D^{\lambda, \mu}}(E)=d_{\mu} \cdot E_{\mathbf{a}}$. It follows that $d_{\mu}$ is equal to $2^{e}$ where $e$ is the number of essential boxes. Using Corollary 5.6, one gets:

Proposition 7.11. A D-box $\mathfrak{a}$ is essential if and only if it is the highest staircase box in a non-related component.

(Note that this result covers also the extremal component of the following type:

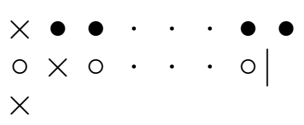

By "|" we indicate the end of a row.)

If the highest and leftmost box from the proposition lies in column $h$ where $h<m$, then one gets multiplicity 2 by applying $\partial_{h}$ to $E_{\mathbf{b}}$ where $\mathbf{b}=(\ldots,-1,1, \ldots)$, the displayed entries being in the $(h+1)$ st and $h$ th places. If the component is of the above form, then we get multiplicity 2 by applying $\partial_{m}$ to $E_{\mathbf{c}}$ where $\mathbf{c}=(1,1, \ldots)$.

7.2. Case 2: $\lambda$ is of type 2 and $\mu$ is of type 1

LEMMA 7.12. Exactly one s-operation supplies $v_{\alpha}$ with a bar. 
Proof. Suppose not. Then $v_{\alpha}$ receives a bar at least twice, so the $\bar{v} / v$ ribbon meets column $m$ at least three times. Consider the relevant (bottom) part of the $\bar{v} / v$-ribbon depicted below:

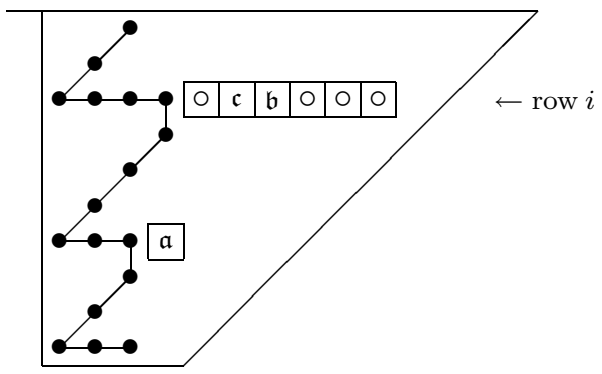

We shall use Proposition 5.5 three times. The box $\mathfrak{a}$ here is a $\sim D$-box by the separation property. We claim that all the boxes in row $i$, to the right of the depicted segment of the $\bar{v} / v$-ribbon, are actually $\sim D$-boxes. Suppose otherwise and let $\mathfrak{b}$ be the leftmost such $D$-box. Then its left neighbor $\mathfrak{c}$ is a $\sim D$-box by the separation property. Suppose first that $\mathfrak{b}$ is a $z$-box. Then the box just above $\mathfrak{b}$ is a $\sim D$-box by Lemma 3.10. Note that "a zero comes from this last box to $\mathfrak{c}$ from above, the $\bar{v} / v$-ribbon brings another zero which comes to $\mathfrak{c}$ from the left", and $\mathfrak{c}$ causes vanishing - a contradiction.

Suppose now that $\mathfrak{b}$ is a $v$-box. Then "its $v$-ribbon brings a zero to $\mathfrak{c}$ from above, the $\bar{v} / v$-ribbon brings another zero which comes to $\mathfrak{c}$ from the left", and again, $\mathfrak{c}$ causes vanishing - a contradiction.

Finally, picking the $\sim D$-box over $\mathfrak{a}$ in row $i$, we see that "a zero comes from this box to $\mathfrak{a}$ from above, the $\bar{v} / v$-ribbon brings another zero that comes to $\mathfrak{a}$ from the left", and $\mathfrak{a}$ causes vanishing. We get a contradiction.

Lemma 7.13. One has $i\left(\mu^{b}\right)-i\left(\lambda^{b}\right) \leq 1$.

The proof of this lemma is identical to the one of Lemma 7.3. (Note that $\mathfrak{d}$ from this last proof is a $\sim D$-box because $\mu$ is of type 1.)

We know from Section 3 that only $z$ 's and $v_{\alpha}$ receive a bar, and each element once (cf. Lemma 7.12). Suppose that

$$
z_{1}<\ldots<z_{p}<v_{\alpha}<z_{p+1}<\ldots<z_{k} .
$$

We now show that $D_{\lambda}^{b} \subset D_{\mu}^{b}$ (the discussion ending with Corollary 7.15 will also apply to Case 4). As a consequence of the properties of reduced decompositions of $w_{\lambda}$ from Lemma 3.1 and Proposition 3.2, we get the following fact (here, the non-vanishing assumption is not necessary):

LEMMA 7.14. If $v_{\alpha}$ receives a bar exactly once then it receives it together with some $z_{i}, i \leq p$, if $p$ is odd, and together with some $z_{i}, i \leq p+1$, if $p$ is even. 
Proof. We illustrate the principle of the proof in the following two situations (the reader can easily reconstruct the formal proof from that).

Let $z_{1}<v_{\alpha}<z_{2}<z_{3}$. If $v_{\alpha}$ receives a bar together with $z_{3}$ then two transpositions of $\left(v_{\alpha}<z_{2}\right)$ are needed. If $v_{\alpha}$ receives a bar together with $z_{2}$, and $z_{1}$ and $z_{3}$ got bars before, then two transpositions of $\left(z_{1}<z_{2}\right)$ are needed. If $v_{\alpha}$ receives a bar together with $z_{2}$, and $z_{1}$ and $z_{3}$ will get bars later, then two transpositions of $\left(z_{2}<z_{3}\right)$ are needed.

Suppose now that $z_{1}<z_{2}<v_{\alpha}<z_{3}<z_{4}$. If $v_{\alpha}$ receives a bar together with $z_{4}$ then two transpositions of $\left(v_{\alpha}<z_{3}\right)$ are needed.

In all cases we get a contradiction with Lemma 3.1 or Proposition 3.2. Also in general, assuming that the assertion of the lemma is not true, we deduce that the length of $w_{\lambda}$ should be larger than it actually is.

For the sake of the next two results, let the distinguished row be the bottom row of $D$ where the $v_{\alpha}$-ribbon meets column $m$. Note that if the number of this row is $q$ then this means that for $q$ odd, $v_{\alpha}$ receives a bar together with $z_{q}$, and for $q$ even, $v_{\alpha}$ receives a bar together with $z_{q+1}$. Consider the $v_{\alpha}$-ribbon. If we increase the segment of $v_{\alpha}$-boxes in the distinguished row by a line segment whose length is the sum of the lengths of all segments of pure $v_{\alpha}$-boxes below the distinguished one, we get a subset encoding the same reduced decomposition. (We must remember that there are also non-pure $v_{\alpha}$-boxes appearing as an antidiagonal line segment starting from the rightmost pure $v_{\alpha}$-box in the distinguished row and ending in the box $(p+1$, $\left.m+1-v_{\alpha}\right)$.) It is clear that by inverting the operations of breaking rows and by straightening the $v_{\alpha}$-ribbon to the distinguished row (the operation just described), we obtain a diagram $D^{\prime}\left({ }^{4}\right)$, whose bottom part is still contained in $D_{\mu}^{b}$, in which all $z$-ribbons and the pure boxes of the $v_{\alpha}$-ribbon appear as consecutive rows. (Using the $q$ introduced above, we note that the length of row $q$ in $D^{\prime}$ can be larger than the length of row $q+1$.) Now we invoke e-transformations from Section 3. It follows that by applying to $D^{\prime}$ a sequence of $p-q$ operations that are inverse to e-transformations, we get precisely the diagram $D_{\lambda}$. This implies, with the above assumptions:

Corollary 7.15. One has $D_{\lambda}^{b} \subset D_{\mu}^{b}$.

Under the non-vanishing assumption, we have the following precise description of the $v_{\alpha}$-ribbon in the present Case 2:

Proposition 7.16. The $v_{\alpha}$-ribbon is the union of two connected line segments. One is an antidiagonal segment starting from $\left(p+1, m+1-v_{\alpha}\right)$

$\left({ }^{4}\right)$ In this subsection (and also in Case 4 below), by a diagram we shall mean a subset of $D_{\mu}$ which is the union of connected subsets of rows of $D_{\mu}$, each starting from the leftmost column. 
and ending in some box in the distinguished row, the second is a horizontal line segment joining this box with the box $(q, m)$.

Proof. Since $\mu$ is of type 1, the part $\mu_{\alpha}^{t}$ is larger than the number of rows in $D_{\mu}^{b}$. Hence, over any box appearing in column $m$ of $D_{\mu}^{b}$ in the distinguished row or below it, there exists a $\sim D$-box (or the rightmost $D$ box) in $\mu_{\alpha}^{t}$. In the picture below, $1,2,3,4,5,6$ are $\sim D$-boxes and $1,2,3,4,5$ lie respectively over $1^{\prime}, 2^{\prime}, 3^{\prime}, 4^{\prime}, 5^{\prime}$.

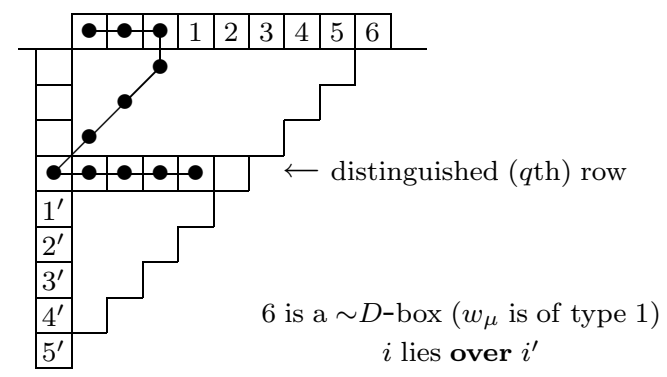

Suppose that the following configurations of boxes $\mathfrak{a}$ and $\mathfrak{b}$ appear in the distinguished row or below it:

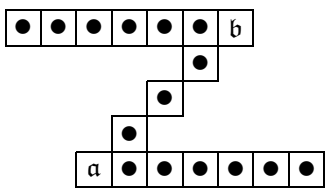

Here $\mathfrak{a}$ and $\mathfrak{b}$ are $\sim D$-boxes. For the distinguished row and the rows below it, we can apply Proposition 5.5. We note that "a zero (at least coming from $1,2,3,4,5,6)$ comes from the left to $\mathfrak{a}$, another zero from $\mathfrak{b}$ comes to $\mathfrak{a}$ from above", and $\mathfrak{a}$ causes vanishing. We get a contradiction.

In particular, we see that the distinguished row is the deformed special $\lambda$-row.

We know that in Case 2, to get the set of $z$-boxes together with the set of pure $v_{\alpha}$-boxes, we take $D_{\lambda}^{b} \subset D_{\mu}^{b}$ and perform some e-transformations followed by some operations of breaking $z$-ribbons. In fact, in this process, one must perform first all possible e-transformations, and then the maximal deformation. This follows from the following two facts.

Proposition 7.17. To avoid the vanishing $\partial_{\mu}^{D}(E)=0$, any time an e-transformation is possible, one must perform it.

Proof. Recall that no push down operation can be applied here by Lemma 7.13. Suppose that an e-transformation is possible and we do not make it: 

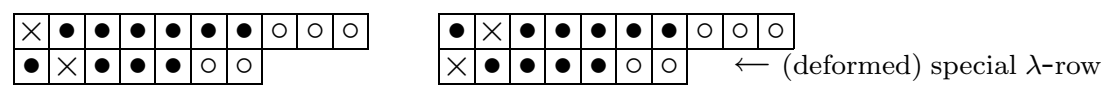

Then, whatever modification we apply, we get the following configuration of two $\sim D$-boxes $\mathfrak{a}$ and $\mathfrak{b}$ :
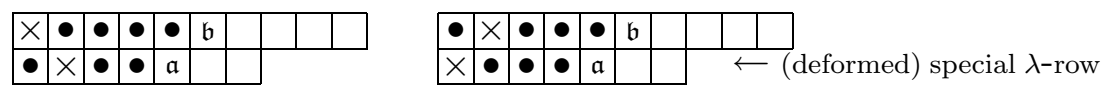

We now use Proposition 5.5 in the following way. Since "the $\bar{v} / v$-ribbon brings a zero that comes to $\mathfrak{a}$ from the left and another zero comes from $\mathfrak{b}$ to $\mathfrak{a}$ from above", $\mathfrak{a}$ causes vanishing - a contradiction.

Denoting by $D^{\prime}$ the diagram obtained by applying all possible e-transformations to $D_{\lambda}^{b} \subset D_{\mu}^{b}$, and arguing in a similar way to the proof of Lemma 7.2, we infer the following result:

Proposition 7.18. The subset of z-boxes together with the set of pure $v_{\alpha}$-boxes is the effect of applying the maximal deformation to the diagram $D^{\prime}$.

We can now examine the components (i.e. the connected components of $\left.D_{\mu}^{b} \backslash D_{\lambda}^{b}\right)$.

Proposition 7.19. The components above the special $\lambda$-row form a $3 / 2$ strip. The special component is a 1-strip with lowest boxes in the special $\lambda$-row. All components between the special one and the extremal one form a 1/2-strip. The extremal component is an almost 3/2-strip whose 3/2-strip is a connected line segment situated in the row of the highest box of the proper 2-strip.

Proof. For all components above the special one the proof is the same as that of [PR2, Proposition 6.7].

To prove the remaining assertions, we shall use the first picture from the proof of Proposition 7.16. Supposing either the components below the special $\lambda$-row in $D_{\mu}^{b} \backslash D_{\lambda}^{b}$ do not form a 1/2-strip, or the extremal component is not of the desired form, whatever deformation we make, we get the following configuration of two $\sim D$-boxes below the special row:

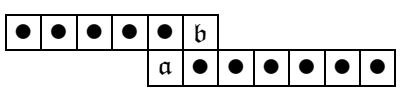

For the rows below the special one, we can apply Proposition 5.5. We note that "a zero (at least coming from 1,2,3,4,5,6) comes to a from the left, another zero comes from $\mathfrak{b}$ to $\mathfrak{a}$ from above", and $\mathfrak{a}$ causes vanishing-a contradiction.

It remains to analyze the special component as in the picture below (the component is shown before deformations): 


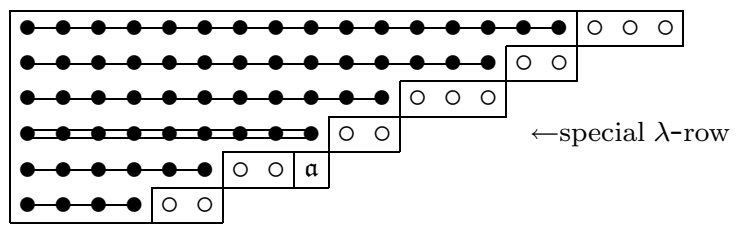

Suppose that $\mathfrak{a}$ is a $\sim D$-box. After performing all the possible e-transformations we get

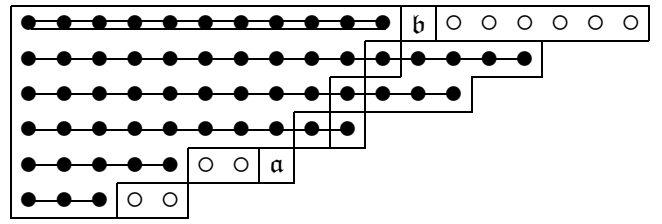

From the above we know that no other deformations of rows below the present special one can occur. We now use Proposition 5.5 in the following way. "A zero (coming at least from $1,2,3,4,5,6$ ) comes from the left to $\mathfrak{a}$, another zero from $\mathfrak{b}$ comes to $\mathfrak{a}$ from above", and $\mathfrak{a}$ causes vanishing - a contradiction.

Translating this into initial shape-data, we see that the lowest boxes in the special component appear in the special $\lambda$-row. A similar argument shows that the special component cannot have two boxes in one column.

We have a complete description of the connected components of $D_{\mu}^{b} \backslash D_{\lambda}^{b}$. For the components appearing above the special one, the results (a)-(e) given before Proposition 7.7 hold true.

It follows from Proposition 7.18 that the set of $z$-boxes together with the set of pure $v_{\alpha}$-boxes is uniquely determined if $\partial_{\mu}^{D}(E) \neq 0$ and $r_{D} \in$ $R\left(w_{\lambda}\right)$.

Moreover, there are no $v$-boxes other than $v_{\alpha}$-boxes in the special component and below it. Indeed, suppose otherwise and pick the highest and leftmost such $v$-box. Then its left neighbor must be a $\sim D$-box by the separation property. This $\sim D$-box causes vanishing by Proposition 5.5 because "a zero comes to it from the left and from above", since the $v$-box in question lies under the rightmost box of a row from the top part.

Thus, arguing as in the proof of [PR2, Theorem 8.1], we get the following result.

Proposition 7.20. There exists at most one $D \subset D_{\mu}$ such that $\partial_{\mu}^{D}(E)$ $\neq 0$ and $r_{D} \in R\left(w_{\lambda}\right)$.

Denote this unique $D$ by $D^{\lambda, \mu}$. Here is a way to construct it (cf. [Exx, 5]). 
RECIPE 7.21. Let $\lambda, \mu$ be two shapes satisfying the conditions:

(a) $D_{\mu}^{b} \supset D_{\lambda}^{b}, D_{\mu}^{b} \backslash D_{\lambda}^{b}$ is a 3/2-strip and $\stackrel{\circ}{l}\left(\mu^{b}\right)-\stackrel{\circ}{l}\left(\lambda^{b}\right) \leq 1$.

(b) The $\lambda$-part of at most one row from the top part ends over a component.

The recipe is:

(i) Apply the e-transformation to $D_{\lambda}$ as many times as possible to obtain a diagram $D^{\prime}$.

(ii) Apply the maximal deformation to $D^{\prime}$ to obtain a set $D^{\prime \prime}$.

(iii) Shift the bottom part of the diagram $D_{\mu}^{b}$ together with $D^{\prime \prime}$. For every (shifted deformed) component of $D_{\mu}^{b}$ pick the row of $D_{\lambda}^{t}$ which ends over the component. Subtract the segment of the row which ends over the roof of the component, and push it down to the roof.

LEMma 7.22 (cf. [PR2, Lemma 8.5]). $D_{\mu}^{t} \backslash D_{\lambda}^{t}$ is a 1/2-strip and no $(\mu-\lambda)$-box lies over the staircase of a related component.

We complement the definition of the compatibility of $\lambda$ and $\mu$ by adding the following requirements.

(C2.1) The special component is a 1-strip with lowest boxes in the special $\lambda$-row. All components between the special one and the extremal one form a 1/2-strip. The extremal component is an almost 3/2strip whose $3 / 2$-strip is a connected line segment situated in the row of the highest box of the proper 2-strip. Neither the special component nor the components below it are related.

Proposition 7.23. $\partial_{\mu}^{D^{\lambda, \mu}}(E) \neq 0$ if and only if $\mu$ is compatible with $\lambda$.

Proof. We can almost repeat the proof of Proposition 7.10. The main difference between the definitions of compatibility in Cases 2 and 1 is the addition, to Definition 6.1(i)(ii), of the conditions for the special component and for the components lying below it. These modifications are necessary by Proposition 7.19. The only thing which has to be proved is that conditions (i)-(v) of Definition 6.1 imply the non-existence of bad boxes in the special component and in the components below it. But this actually follows from Definition 6.1(iii) by Proposition 5.5. ("No zero can come to such a box from above.")

Proposition 7.24. One has $d_{\mu}=2^{e}$, where $e$ is the cardinality of the set of non-related components above the special component, with no $(\mu-\lambda)$-boxes over them.

Proof. Each essential box contributes a factor of 2 to the multiplicity $d_{\mu}$. Essential boxes are the highest staircase boxes in non-related components, with no $(\mu-\lambda)$-box over them. No essential boxes can appear in the special 
component and below it by Proposition 5.5. ("There is always a zero coming to such a box from the left.")

\subsection{Case 3: $\lambda$ is of type 1 and $\mu$ is of type 2}

Lemma 7.25. The $\bar{v} / v$-ribbon meets column $m$ no more than twice.

Proof. Suppose otherwise. Then the $\bar{v} / v$-ribbon meets column $m$ at least four times because $w_{\lambda}$ is of type 1 and its $v_{\alpha}$ has no bar. Consider the following schematic picture of the $\bar{v} / v$-ribbon:

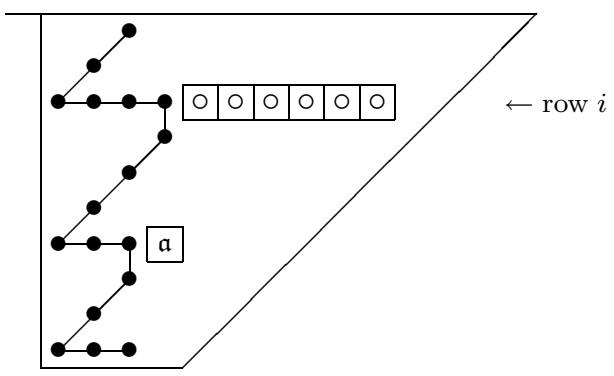

Let $i$ be the number of the highest row where the $\bar{v} / v$-ribbon meets column $m$. We claim that apart from the segment of $\bar{v}$-boxes, the $i$ th row consists of $\sim D$-boxes. Indeed, assume that $\mathfrak{b}$ is the leftmost $z$-box in row $i$. Then its left neighbor $\mathfrak{c}$ and the box $\mathfrak{d}$ directly above $\mathfrak{b}$ (in the column of $\mathfrak{b}$ ) are $\sim D$-boxes. We use Proposition 5.5 in the following way. "A zero comes from $\mathfrak{d}$ to $\mathfrak{c}$ from above, the $\bar{v} / v$-ribbon brings a zero to $\mathfrak{c}$ from the left", and $\mathfrak{c}$ causes vanishing. Assume now that $\mathfrak{b}$ is the leftmost $v$-box in the $i$ th row with mark $\neq v_{\alpha}$. Let $\mathfrak{c}$ be the left neighbor of $\mathfrak{b}$. Then "the $v$-ribbon brings to $\mathfrak{c}$ a zero from above, the $\bar{v} / v$-ribbon brings a zero to $\mathfrak{c}$ from the left", and $\mathfrak{c}$ causes vanishing. We get a contradiction.

Note that the $\sim D$-box $\mathfrak{a}$ must exist by the separation property and because $D_{\mu}^{b}$ is the diagram of a strict partition. Finally we use Proposition 5.5 in the following way. "A zero comes from the $i$ th row to $\mathfrak{a}$ from above, the $\bar{v} / v$-ribbon brings a zero to $\mathfrak{a}$ from the left", and $\mathfrak{a}$ causes vanishing - a contradiction.

Now, let us consider separately the following two situations. Firstly, assume that $v_{\alpha}$ receives a bar by some $s$-operation.

Proposition 7.26. If the $\bar{v} / v$-ribbon meets column $m$ twice, then the positions of $z$-boxes in $D_{\mu}^{b}$ are uniquely determined.

Proof. Assume that the $\bar{v} / v$-ribbon meets column $m$ twice. This means that $v_{\alpha}$ receives and then loses a bar. This is depicted below: 


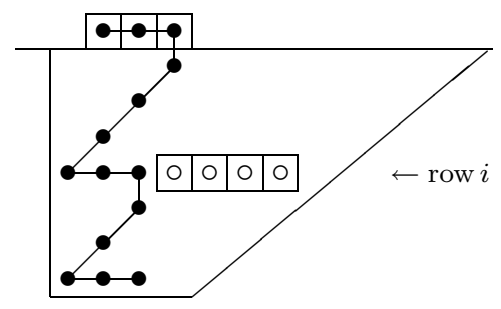

where $i$ is the number of the highest row where the $\bar{v} / v$-ribbon meets column $m$. We know from the proof of the previous lemma that apart from the segment of $\bar{v}$-boxes, row $i$ consists of $\sim D$-boxes. Let us introduce the following notation:

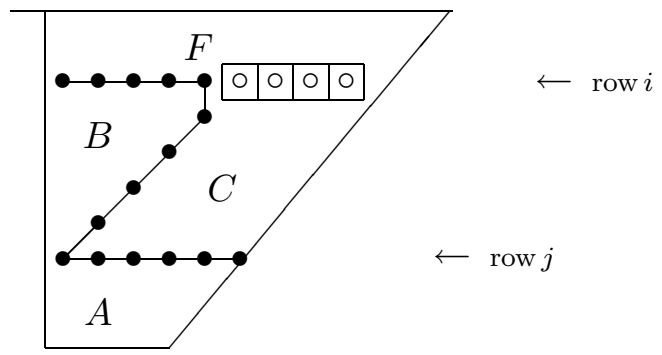

where $j$ is the number of the lowest row where the $\bar{v} / v$-ribbon meets column $m$. We claim that the $j$ th row of $D_{\mu}^{b}$ is entirely filled up by the boxes of this ribbon. Indeed, assume that there is a $\sim D$-box $\mathfrak{a}$ in this row. We use Proposition 5.5 in the following way: "A zero comes from the $i$ th row to $\mathfrak{a}$ from above, the $\bar{v} / v$-ribbon brings another zero to $\mathfrak{a}$ from the left", and $\mathfrak{a}$ causes vanishing - a contradiction. Thus by the connectedness or separation property our claim follows.

There are no $\sim D$-boxes in the area marked by $A$. Indeed, asume that there exists a $\sim D$-box $\mathfrak{a}$ there. We use Proposition 5.5 in the following way. "A zero comes from the $i$ th row to $\mathfrak{a}$ from above, another zero comes to $\mathfrak{a}$ from the left", and $\mathfrak{a}$ causes vanishing - a contradiction. Thus $A$ is filled up entirely by horizontal line $z$-ribbons.

Note that no $\sim D$-box appears over the $\sim D$-boxes of row $i$ (otherwise we get vanishing). Hence a connected line segment of $D$-boxes must appear in each bottom row over the part of row $i$ consisting of $\sim D$-boxes.

It follows from the properties of $v$-ribbons that no $v$-box can lie in $B$. Also, it follows either by the properties of $z$-ribbons (for $B$ ), or from the last-mentioned property (for $C$ ) that no $z$-ribbon starting in $F$ can have its continuation in the union $B \& C$ consisting of $B, C$, and the displayed antidiagonal line segment of non-pure $v_{\alpha}$-boxes between them.

Therefore a family of $z$-ribbons must be contained entirely in the area $B \& C$. 
In $C$ no pair of neighbor boxes situated in one row can appear because of the $D$-boxes in row $i$ (otherwise we get vanishing). So we exclude such a situation; in particular it is impossible that a row consisting entirely of $\sim D$-boxes appears in $B \& C$.

Note that the displayed non-pure $v_{\alpha}$-boxes in $B \& C$ must be $z$-boxes by Proposition 3.2 (an $s$-operation which moves $v_{\alpha}$ backward must move some $z$ forward). These $z$-boxes must have different marks and since they cannot have their continuation in $F$, they must start in the leftmost boxes in $B$. Consequently, $B$ consists of horizontal segments of $z$-boxes only.

Let us analyse the successive $z$-ribbons in $B \& C$, starting from the bottom one. Suppose that some of them has a breaking point; pick the lowest such breaking box $\mathfrak{a}$. By the above, it must belong to $C$. Then by the properties of $z$-ribbons we get a "staircase" of breaking boxes starting with $\mathfrak{a}$ and ending with a "roof" which is a line segment of $\sim D$-boxes ending with the rightmost box of $D_{\mu}^{b}$ in this row. The only possibility to avoid vanishing is to fill up this roof with some pure $v$-boxes with mark smaller than $\alpha$. This is done by pushing forward a final part of some top row. But then we use Proposition 5.5 in the following way: "A zero comes from the last box of the remaining part of the just mentioned related row to a $\sim D$-box in row $i$, another zero comes to this box from the left", and this box causes vanishinga contradiction. Therefore also $B \& C$ consists of a family of horizontal line segments ending, perhaps, with a single $\sim D$-box at the rightmost places.

We finally analyze $F$. First we remark that obviously no row consisting entirely of $\sim D$-boxes can appear there. Next we note that the non-pure $\bar{v}$-boxes in $F$ displayed in the last but one picture are $z$-boxes by Proposition 3.2. Hence they belong to $z$-ribbons starting in consecutive leftmost boxes of $F$. They cannot have a continuation in the lower marked areas. Assume that some of them can be broken and pick the lowest such box $\mathfrak{a}$. Then by the properties of $z$-ribbons we get a "staircase" of breaking boxes starting with $\mathfrak{a}$ and ending with a "roof" which is a line segment of $\sim D$-boxes ending with the rightmost box of $D_{\mu}^{b}$ in this row. The only possibility to avoid vanishing is to fill up this roof with some pure $v$-boxes with mark smaller than $\alpha$. This is done by pushing forward a final part of some top row. But then the length of the $z$-ribbon starting in the row of $\mathfrak{a}$ is at most equal to the length of the $z$-ribbon which is immediately below it - a contradiction. We conclude that over the $i$ th row, $F$ consists of horizontal line segments of $z$-ribbons.

What can happen in the remaining part of $F$ can be analyzed exactly in the same way as in Case 1: the components are deformed 3/2-strips having $v$-boxes in their roofs, if related.

COROLlary 7.27. Under the assumption of the proposition, $\stackrel{i}{l}\left(\mu^{b}\right)-i\left(\lambda^{b}\right)$ $=2$. 
We can now invoke the special $\mu$-row appearing in or below row $i$. The single rightmost $\sim D$-boxes appearing in the last proof must lie below the special $\mu$-row. Indeed, if such a box $\mathfrak{a}$ appears between row $i$ and the special $\mu$-row, then we use Proposition 5.5 in the following way: "A zero comes from row $i$ to $\mathfrak{a}$ from above, another zero comes to $\mathfrak{a}$ from the left", and $\mathfrak{a}$ causes vanishing - a contradiction. We may picture the part of $D_{\mu}^{b}$ below the $i$ th row as follows:

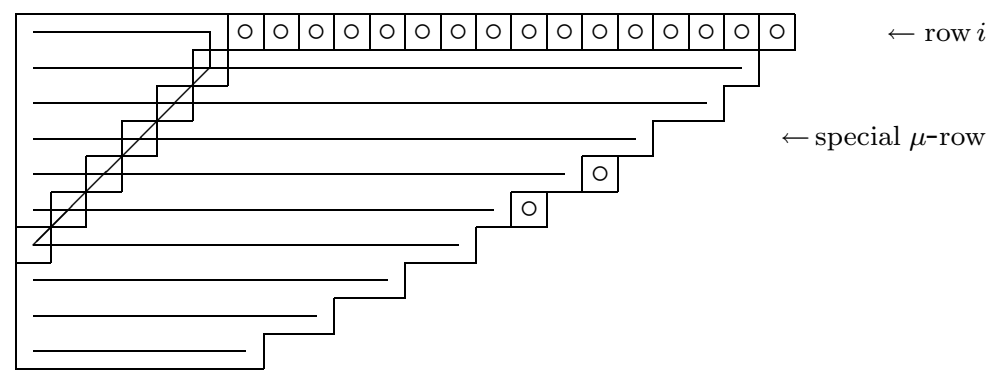

We translate these conditions into the initial shape-data. For the moment we shall be concerned only with the displayed part of $D_{\mu}^{b}$, for simplicity. Let us remove from the above picture all $D$-boxes with a pure $v$-mark or pure $\bar{v}$-mark. The lengths of the remaining $z$-ribbons, depicted as lines, are equal to the lengths of the rows of the corresponding part of $D_{\lambda}^{b}$ :

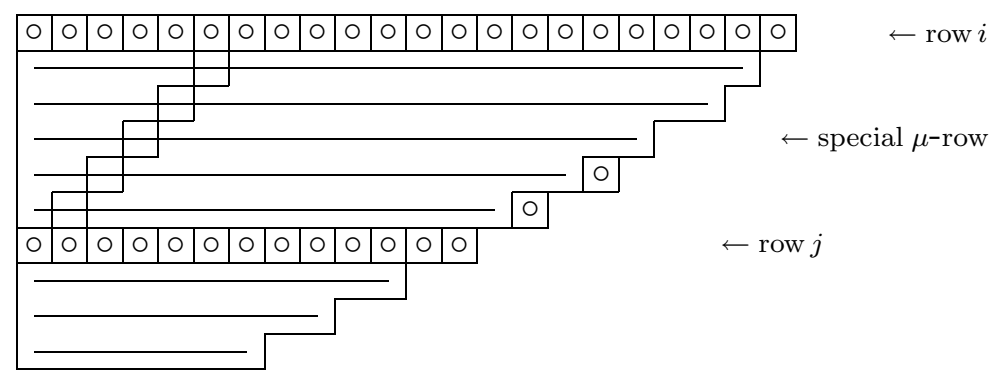

This configuration of boxes encodes the same reduced decomposition as this part of $D_{\lambda}^{b}$ with the following 2-strip added:

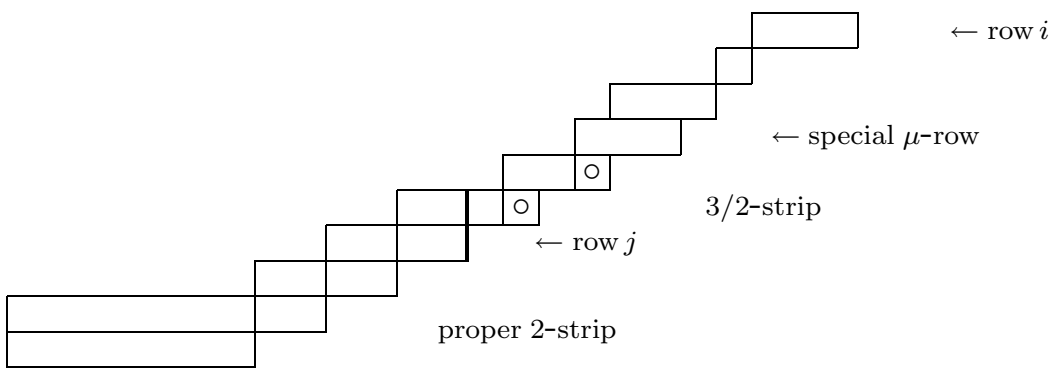


Here row $i$ is the highest row of the 2-strip and row $j$ is the row of the lower 1-strip of the proper 2 -strip. The single rightmost $\sim D$-boxes form the excrescence of the $3 / 2$-strip; this excrescence is a degenerate strip. They appear between the special $\mu$-row and the lower 1-strip of the proper 2strip. After applying the maximal deformation to this part of $D_{\lambda}^{b}$ with the 2 -strip, we get the configuration in the last but one picture. We see that the appropriate boxes of the $\bar{v} / v$-ribbon fill up properly the places now occupied by $\sim D$-boxes (cf. Recipe 7.29 (iii) below) only if the following condition is satisfied:

" $\lambda_{\alpha}^{t}$ ends over the rightmost box of the lower 1-strip of the proper 2-strip of the extremal component."

The same arguments as in Case 1 determine the remaining components of $D$. We can summarize this discussion in the following:

Proposition 7.28. There exists a unique $D \subset D_{\mu}^{b}$ such that $r_{D} \in$ $R\left(w_{\lambda}\right)$ and $\partial_{\mu}^{D}(E) \neq 0$ and whose $\bar{v} / v$-ribbon meets column $m$ exactly twice.

The set $D$ from the proposition is constructed in the following way (cf. $[\operatorname{Exx}, 6])$.

RECIPE 7.29. The assumptions are as in Recipe 7.8 with the following modification: the extremal component is a 2-strip meeting the special $\mu$-row; $\lambda_{\alpha}^{t}$ ends over the rightmost box of the lower 1-strip of the proper 2-strip. The modifications of all non-extremal components are as in Recipe 7.8. The extremal component is deformed as follows:

(i) Push down all the bottom rows of $D_{\lambda}^{b}$, up to the highest one of the 2-strip.

(ii) Lower by one row the positions of the next rows of $D_{\lambda}^{b}$, up to the highest one in the component.

(iii) The lower 1-strip of the proper 2-strip and the highest row of the component are not occupied by D-boxes now. Inscribe in these two rows the boxes of the $\bar{v} / v$-ribbon in such a way that the former row is filled up entirely.

Conversely, it is not hard to show, with the help of Proposition 5.5 and Corollary 5.6, that the special (= extremal) component contributes a factor of 1 to the multiplicity $d_{\mu}$.

Now we examine the case when the $\bar{v} / v$-ribbon does not meet column $m$. This case is very much similar to Case 1 when $\lambda$ and $\mu$ are of type 1 . In fact, arguing in the same way as in Case 1 we show the following result.

Lemma 7.30. The set of $z$-boxes is the maximal deformation of $D_{\lambda}^{b}$ in $D_{\mu}^{b}$, and the $z$-boxes with the same mark can appear in at most two successive rows. 
In the present subCase 3 we have:

Proposition 7.31. One has $\stackrel{\circ}{l}\left(\mu^{b}\right)-\stackrel{\circ}{l}\left(\lambda^{b}\right)=2$ or 1 .

Proof. The inequality $\stackrel{i}{l}\left(\mu^{b}\right)-\stackrel{\circ}{l}\left(\lambda^{b}\right)>2$ means that there exist at least three rows with boxes in $D_{\mu}^{b} \backslash D_{\lambda}^{b}$. We know from Lemma 7.30 that, to avoid vanishing, we must perform the maximal deformation of $D_{\lambda}^{b}$ in $D_{\mu}^{b}$. After the maximal deformation, we get one of the two configurations of boxes pictured below:

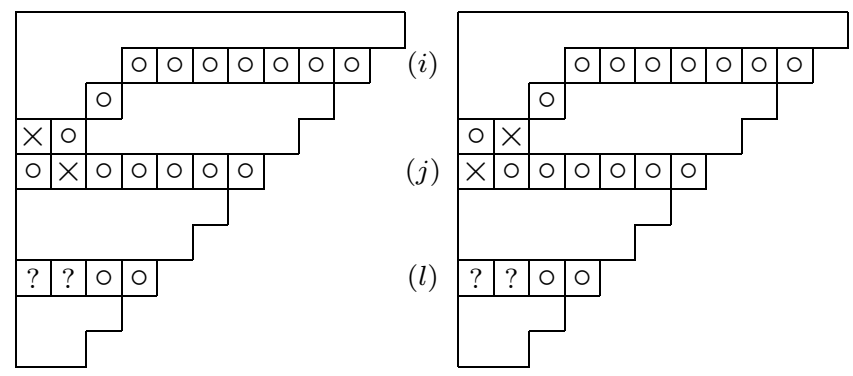

It follows from the properties of $v$-ribbons that $v$-boxes with mark $\alpha$ can appear in row $i$ only. But then rows $j$ and $l$ consist of $\sim D$-boxes (no $v$-boxes can appear there) and we get vanishing - a contradiction.

Now suppose that $\stackrel{l}{l}\left(\mu^{b}\right)=\stackrel{i}{l}\left(\lambda^{b}\right)$. In this case, there is no push down operation in the maximal deformation. Since $\lambda$ is of type 1 and $\mu$ is of type $2, \mu_{\alpha}^{t}$ is exceptional. The partitions $\mu^{b}$ and $\lambda^{b}$ have equal lengths and there is no component of $D_{\mu}^{b}$ over which this row ends. Hence it is impossible to obtain $D \subset D_{\mu}$ satisfying $r_{D} \in R\left(w_{\lambda}\right)$ if $\stackrel{\circ}{l}\left(\mu^{b}\right)=\stackrel{\circ}{l}\left(\lambda^{b}\right)$.

Once again we see that we get the (deformed) extremal component by adding to $D_{\lambda}^{b}$ a 2-strip and performing the maximal deformation. Note that

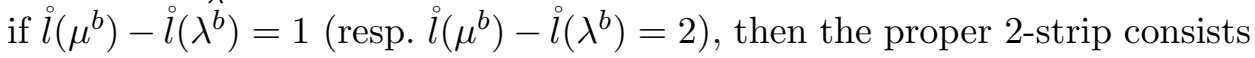
of 1 column (resp. of $\geq 2$ columns).

We see that as in the former subCase 3 , the excrescence of the $3 / 2$-strip must appear between the special $\mu$-row and the lower 1-strip of the proper 2strip, and forms a degenerate strip. On the other hand to eliminate a possible vanishing caused by boxes from the present lowest row with $\sim D$-boxes, the following condition must be satisfied:

"If $\lambda_{\alpha}^{t}$ ends over a box $(a, b)$ of the extremal component with $b<m-1$, then it ends over the rightmost box of the lower 1-strip of the proper 2-strip, or more to the right."

Arguing as in the proof of [PR2, Theorem 8.1], one shows:

Proposition 7.32. There is at most one $D \subset D_{\mu}$ such that $r_{D} \in R\left(w_{\lambda}\right)$ and $\partial_{\mu}^{D}(E) \neq 0$ and whose $v_{\alpha}$-ribbon does not meet column $m$. 
It is clear that the set $D$ from this proposition is obtained by applying Recipe 7.8 to a pair of shapes $\lambda, \mu$ for which the following conditions hold:

(1) $D_{\mu}^{b} \supset D_{\lambda}^{b}$; the extremal component meets the special $\mu$-row and forms a 2-strip; the remaining components of $D_{\mu}^{b} \backslash D_{\lambda}^{b}$ are 3/2-strips.

(2) At most one row from $D_{\lambda}^{t}$ ends over a component, and $\lambda_{\alpha}^{t}$ and the extremal component are related (if they are related) in the way explained above.

We note that the assumptions of the two subcases of Case 3 have as their "common part" the following condition:

(*) $\quad \stackrel{i}{l}\left(\mu^{b}\right)-i\left(\lambda^{b}\right)=2$ and $\lambda_{\alpha}^{t}$ ends over the rightmost box of the lower 1-strip of the proper 2 -strip, situated in the column with number $<m-1$.

Therefore in this situation we can apply both Recipes 7.29 and 7.8, which lead, in general, to different $D$ 's. We have, however, the following result:

Proposition 7.33. Assuming $(*)$, there exists at most one $D$ for which $r_{D} \in R\left(w_{\lambda}\right)$ and $\partial_{\mu}^{D}(E) \neq 0$.

Proof. Assuming (*) and applying Recipe 7.29, we get the following configuration of boxes:

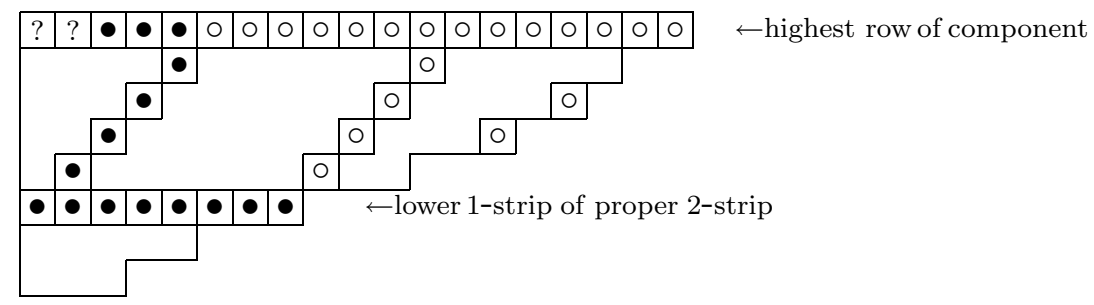

Therefore, if we apply Recipe 7.8 we get the following configuration of boxes:

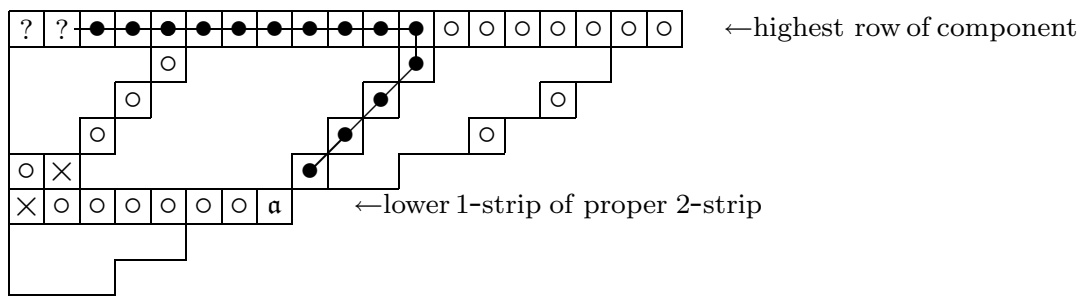




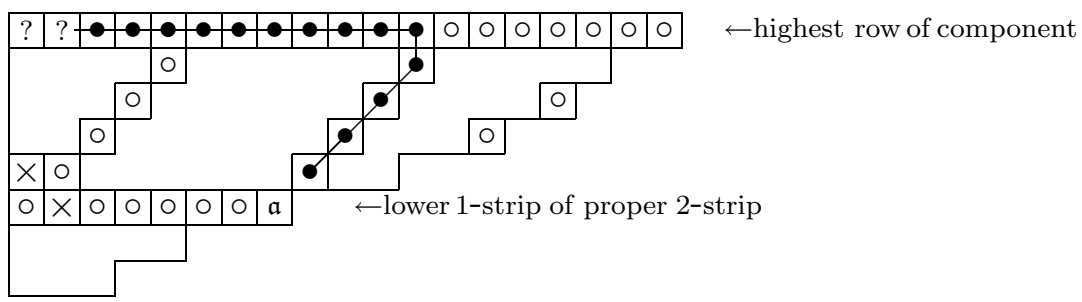

where $\mathfrak{a}$ is a $\sim D$-box. We use Proposition 5.5 in the following way: "the $v_{\alpha}$-ribbon brings to $\mathfrak{a}$ a zero from above, another zero comes to $\mathfrak{a}$ from the left", and $\mathfrak{a}$ causes vanishing. This implies the assertion of the proposition.

We complement the definition of the compatibility of $\lambda$ and $\mu$ by adding the following requirements.

(C1.2) The special component is extremal, and is a 2-strip. If $\lambda_{\alpha}^{t}$ ends over a box $(a, b<m-1)$ of the extremal component, then it ends over the rightmost box of the lower 1-strip of the proper 2-strip, or more to the right. The excrescence of the $3 / 2$-strip of the special component appears between the special $\mu$-row and the lower row of the proper 2-strip, and is a degenerate strip.

It follows from our analysis that $d_{\mu}$ is not zero iff $\mu$ and $\lambda$ are compatible.

It is easy to see that the special (= extremal) component contributes a factor of 1 to the multiplicity $d_{\mu}$. Arguing as in Case 1 we get, in both subcases of Case 3 :

Proposition 7.34. For compatible shapes $\mu$ and $\lambda$, one has $d_{\mu}=2^{e}$, where $e$ is the number of non-related components different from the special one, with no $(\mu-\lambda)$-boxes over them.

7.4. Case $4: \lambda$ and $\mu$ are of type 2

Lemma 7.35. The $\bar{v} / v$-ribbon meets column $m$ exactly once.

The proof is similar to the one of Lemma 7.12.

Lemma 7.36. One has $i\left(\mu^{b}\right)-i\left(\lambda^{b}\right) \leq 1$.

Proof. Suppose not. Then repeating the discussion from the beginning of the proof of Lemma 7.3 shows that the extremal component must contain (after the maximal deformation) one of the following two configurations: 

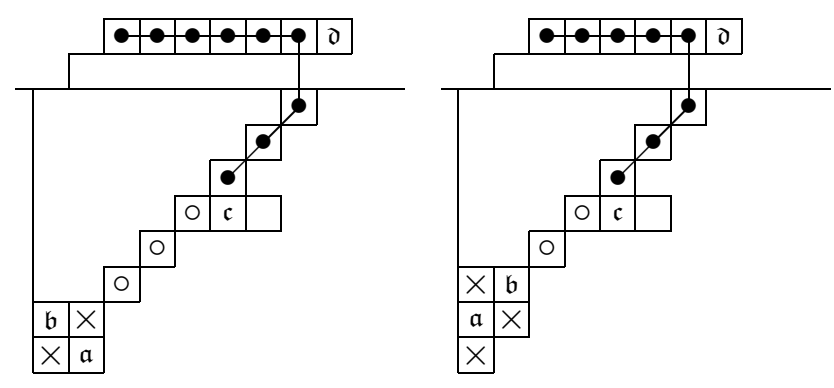

In both, the boxes $\mathfrak{a}$ and $\mathfrak{b}$ are $\sim D$-boxes. The dots indicate boxes from the $v_{\beta}$-ribbon. (If $m=n+1$, then the $\beta$ th row in the top as well as the $v_{\beta}$-ribbon do not exist, and we do not use them in the reasoning.)

Consider first the picture on the left. If $\mathfrak{c}$ is a $\sim D$-box then "a zero comes from $\mathfrak{c}$ to $\mathfrak{a}$ from above, another zero comes from $\mathfrak{b}$ to $\mathfrak{a}$ from the left", and the $\sim D$-box $\mathfrak{a}$ causes vanishing - a contradiction. If $\mathfrak{c}$ is a $D$-box then it must be a $v_{\beta}$-box belonging to the depicted $v_{\beta}$-ribbon. By a property of shape and the separation property, $\mathfrak{d}$ is then a $\sim D$-box. We see that "a zero comes from $\mathfrak{d}$ to $\mathfrak{a}$ from above, another zero comes from $\mathfrak{b}$ to $\mathfrak{a}$ from the left", and $\mathfrak{a}$ causes vanishing - a contradiction.

Consider now the right picture. If $\mathfrak{c}$ is a $\sim D$-box then "a zero comes from $\mathfrak{c}$ to the right neighbor of $\mathfrak{a}$ from above, another zero comes from $\mathfrak{b}$ to $\mathfrak{a}$ from above", and $\mathfrak{a}$ causes vanishing - a contradiction. If $\mathfrak{c}$ is a $D$-box then it must be a $v_{\beta}$-box belonging to the depicted $v_{\beta}$-ribbon. By a property of shape and the separation property, $\mathfrak{d}$ is then a $\sim D$-box. We see that "a zero comes from $\mathfrak{d}$ to the right neighbor of $\mathfrak{a}$ from above, another zero comes from $\mathfrak{b}$ to $\mathfrak{a}$ from above", and $\mathfrak{a}$ causes vanishing. We again get a contradiction.

We can now apply Corollary 7.15 from Case 2, i.e. we know that $D_{\lambda}^{b} \subset$ $D_{\mu}^{b}$, and:

Proposition 7.37. The set of $z$-boxes and pure $v_{\alpha}$-boxes in $D$ is the result of the following operations applied to $D_{\lambda}$ :

(i) Apply the e-transformation as many times as possible and denote the diagram so obtained by $D^{\prime}$.

(ii) Deform the z-boxes of $D^{\prime}$ in the maximal way.

By Lemma 7.36 we know that there is no push down operation in the maximal deformation (ii); in particular we infer that two $z$-boxes with different marks can appear in at most two successive rows.

By the same analysis as in the previous cases or in the proof of [PR2, Proposition 6.7], non-special components are 3/2-strips. We now describe the special component. 
Proposition 7.38. (i) Let $\lambda_{\alpha}^{t}>\mu_{\alpha}^{t}$. Then the special component meets the $\mu$-special row, and is a 3/2-strip. Its excrescence appears between the special $\mu$-row and the special $\lambda$-row and is a degenerate strip.

(ii) Let $\lambda_{\alpha}^{t} \leq \mu_{\alpha}^{t}$. Then the special component is a 1-strip with lowest boxes in the special $\lambda$-row, and the components between the special $\lambda$-row and the special $\mu$-row form a 1/2-strip.

Proof. We show (i). Let us divide the special component into the "upper part" consisting of boxes in the special $\lambda$-row and in the rows above it, and the "lower part" consisting of boxes below the special $\lambda$-row:

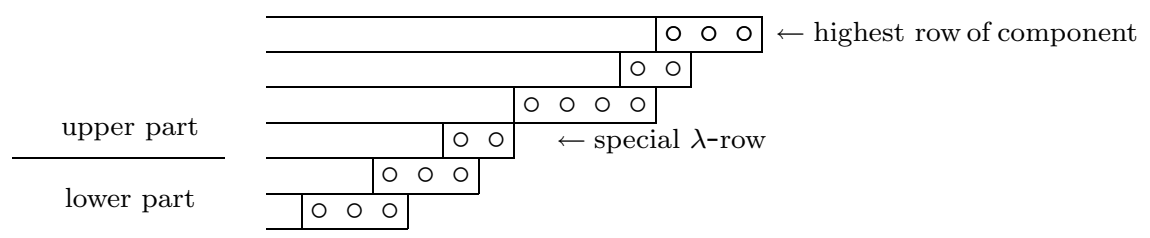

In the following example, " $\square$ " are the boxes of $\mu$, and " $\bullet$ " the dots of $\lambda$ :

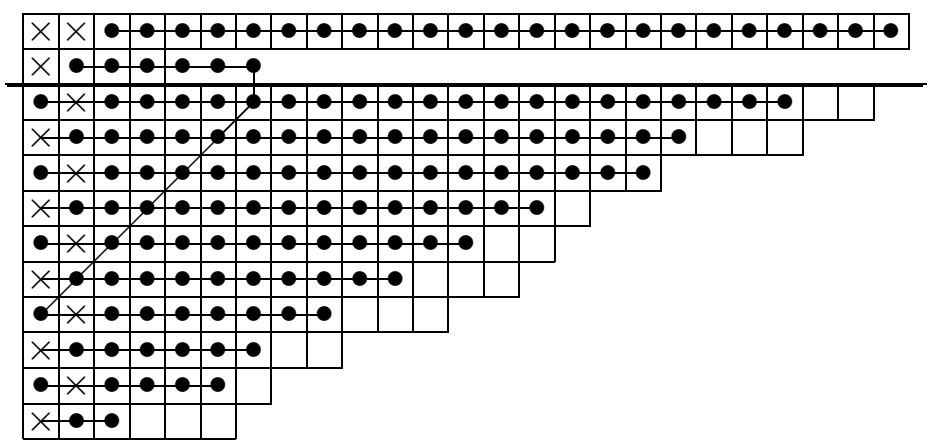

Here row 7 is the special $\lambda$-row. The boxes of the lower part are situated in the last three rows, and the ones of the upper part in the previous four.

Perform all possible e-transformations (this is necessary to avoid vanishing by Proposition 7.17) and then the maximal deformation. In our example, we first perform three e-transformations and then the maximal deformation:

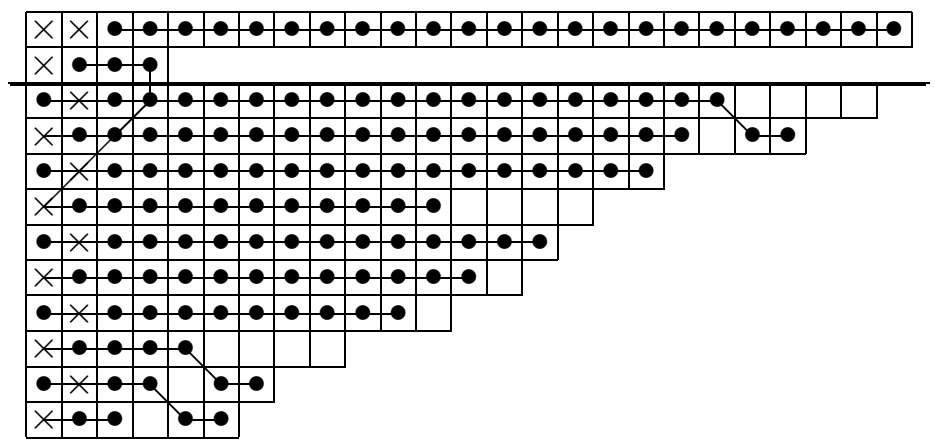


From the lower part, after these deformations we get the roof situated in the row just below the original special $\lambda$-row (row 8 in the last picture). Observe that the deformed special $\lambda$-row (the 4 th dotted row in the last picture) ends over the rightmost box of the roof. Note also that "the deformed $\bar{v} / v$-ribbon brings a zero to the box ending this deformed special $\lambda$-row". The only possibility to avoid vanishing caused by this box and the roof is that we push the appropriate segment of the $v_{\alpha}$-ribbon to fill this roof entirely:

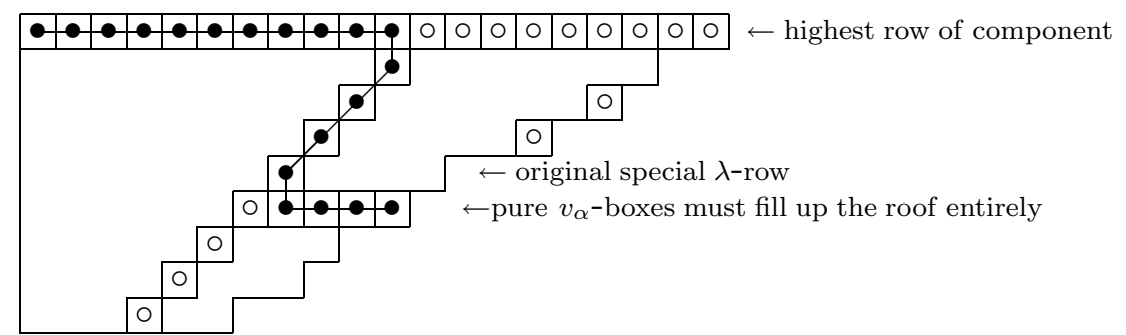

(We cannot avoid vanishing by pushing down from top a line segment of $v$ boxes with mark $<\alpha$, because no transposition between the $v$ 's is possible by Proposition 3.2.)

Using Proposition 5.5, we note the following two facts:

- no $\sim D$-box can appear under this roof;

- no $\sim D$-box can appear in the special $\mu$-row and in the rows above it. (Indeed, to a possible $\sim D$-box in these areas "comes a zero from above and another zero from the left", and such a $\sim D$-box would cause vanishing.)

It follows that the non-deformed special component has the following form:

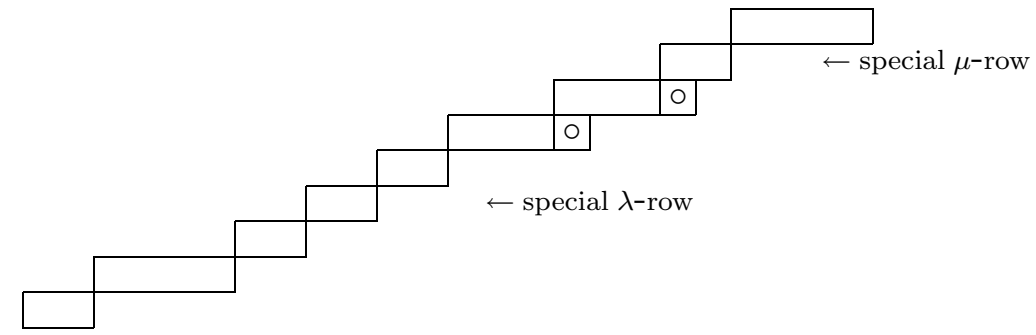

i.e. it is a $3 / 2$-strip whose excrescence, displayed here by the single $\sim D$ boxes, appears between the special rows, and forms a degenerate strip by Proposition 5.5: "for any $\sim D$-box between the special rows, a zero comes to it from above".

The proof of (ii) is very similar to the one in Case 2, and we omit it, giving only the following hint: for any $\sim D$-box between the special rows, "there is a zero coming to it from the left". 
It follows from Propositions 7.37 and 7.38 that the positions of $z$-boxes and pure $v_{\alpha}$-boxes are uniquely determined. Arguing as in the proof of [PR2, Theorem 8.1], one shows that the positions of other $v$-boxes are also uniquely determined. We thus get:

Proposition 7.39. There exists at most one $D$ such that $\partial_{\mu}^{D}(E) \neq 0$ and $r_{D} \in R\left(w_{\lambda}\right)$.

This unique $D$ can be constructed using Recipe 7.21 with the following modification in the case when $\lambda_{\alpha}^{t}>\mu_{\alpha}^{t}$ : after performing the e-transformations and the maximal deformation, we deform the $v_{\alpha}$-ribbon to the roof of the deformed lower part of the special component. The construction of this unique $D$ when $\lambda_{\alpha}^{t} \leq \mu_{\alpha}^{t}$ is analogous to the one in Case 2 .

We complement the definition of the compatibility of $\lambda$ and $\mu$ by adding the following requirements.

Assume first that $\lambda_{\alpha}^{t}>\mu_{\alpha}^{t}$. Then the special component meets the special $\mu$-row and is a $3 / 2$-strip whose excrescence forms a degenerate strip lying between the special $\lambda$-row and the special $\mu$-row. If $\lambda_{\alpha}^{t} \leq \mu_{\alpha}^{t}$, then the special component is a 1-strip with lowest boxes in the special $\lambda$-row. All components between the special $\lambda$-row and the special $\mu$-row form a $1 / 2$-strip. In both cases the extremal component is an almost 3/2-strip and the special component is not related.

We have seen that the conditions of compatibility are necessary to have non-vanishing. It is easy to see, with the help of Proposition 5.5, that they are also sufficient. Moreover, arguing as in the previous cases or in [PR2, Section 9], we get:

Proposition 7.40. In Case 4, the multiplicity $d_{\mu}$ is equal to $2^{e(\lambda, \mu)}$, where for $\lambda_{\alpha}^{t}>\mu_{\alpha}^{t}, e(\lambda, \mu)$ is the number of non-related components different from the special one, with no $(\mu-\lambda)$-boxes over them; and for $\lambda_{\alpha}^{t} \leq \mu_{\alpha}^{t}$, $e(\lambda, \mu)$ is the number of non-related components different from the special one, not lying between the special rows, and with no $(\mu-\lambda)$-boxes over them.

This ends the proof of the theorem.

REMARK 7.41. We discuss what Theorem 6.2 tells us in the case $n=1$. Denote by $a_{i}$, where $i=0,1, \ldots, d-2, d, \ldots, 2 d-2$, the Schubert classes of codimension $i$. In particular, $a_{0}=1, a_{1}=$ [hyperplane] $=: h, a_{2 d-3}=$ [line], and $a_{2 d-2}=$ [point]. Moreover, let $b_{j}$ be the codimension $m-1$ classes of type $j=1,2$. Recall that $a_{i}, i=0, \ldots, d-2$, are of type 1 , and $a_{i}$, $i=d, \ldots, 2 d-2$, are of type 2 (cf. Example 4.4). The theorem asserts: 


$$
\begin{aligned}
a_{i} \cdot h=a_{i+1}, \quad & i=0, \ldots, d-3, \\
a_{i} \cdot h=a_{i+1}, \quad i & =d, \ldots, 2 d-3, \\
a_{d-2} \cdot h & =b_{1}+b_{2}, \\
b_{1} \cdot h & =a_{d}, \\
b_{2} \cdot h & =a_{d} .
\end{aligned}
$$

Equation (3) falls under Case 1 of the theorem: we get multiplicity 1 since there is no component. (4) falls under Case 4 (with $\lambda_{\alpha}^{t}=\mu_{\alpha}^{t}$ ): we get multiplicity 1 since the component is related. (5) falls under Case 1 and the 2 nd subCase 3: the coefficient of $b_{1}$ is 1 since there is no component, and the coefficient of $b_{2}$ is 1 since the component is special. (6) falls under the second subCase 3: we get multiplicity 1 since the component is special. Finally (7) falls under Case 4 (with $\lambda_{\alpha}^{t}<\mu_{\alpha}^{t}$ ): we get multiplicity 1 since there is no component.

8. Concluding remarks. 1. A formula for the product of a Schubert class and $x_{n+1} x_{n+2} \cdots x_{m}$ can be obtained either from Chevalley's multiplication formula $[\mathrm{C}]$ or by the methods of the present paper applied to $E_{(1, \ldots, 1,0, \ldots, 0)}\left(1\right.$ appears $\alpha$ times) instead of $E=E_{(0, \ldots, 0,1, \ldots, 1)}$. The analysis in the former case is much simpler. Note that $x_{n+1} x_{n+2} \cdots x_{m}$ equals $X_{s_{m-1} s_{m-2} \cdots s_{n}}-X_{s_{m} s_{m-2} \cdots s_{n}}$ up to sign, and therefore one has signs in the Schubert class decomposition of the product under consideration.

2. When [PR2] and [PR3] were written, the techniques of orthogonal and symplectic Schubert polynomials were not developed enough to treat the problem of Pieri-type formulas. While revising [PR3], we have intended to keep the spirit of the original article which was a combinatorial analysis of an iterated Leibniz-type formula for divided differences. At present, however, there are formulas presenting Schubert classes in $H^{*}(G)$ as some divided difference operators applied to $\widetilde{Q}$ - and $\widetilde{P}$-polynomials of [PR4]. For example, if $\left(\lambda_{1}^{t}>\ldots>\lambda_{\alpha}^{t}>\lambda_{1}^{b}>\ldots>\lambda_{k}^{b}>0\right)$ is a strict partition then $\sigma\left(\lambda^{t} / / \lambda^{b}\right)$ is evaluated as

$$
\partial_{m} \cdots\left(\partial_{\alpha+2} \cdots \partial_{m}\right) \cdots\left(\partial_{\alpha+1} \cdots \partial_{m-1} \partial_{m}\right) \widetilde{P}_{\left(\lambda^{t}, \lambda^{b}\right)}\left(x_{1}, \ldots, x_{m}\right) .
$$

(Here we consider the odd orthogonal case for simplicity.) In particular, if $m=n+1$ and $a>\lambda_{1}>\ldots>\lambda_{k}>0$, then

$$
\sigma(a / / \lambda)=\partial_{m} \widetilde{P}_{(a, \lambda)}\left(x_{1}, \ldots, x_{m}\right) .
$$

It seems that the algebra of orthogonal and symplectic Schubert polynomials from [PR4], [LP1], and [LP2], combined with the methods of the present paper, should lead to more efficient formulas. 
Added in proof. H. Tamvakis reports (private communication, May 20, 2003) that he, A. S. Buch, and A. Kresch have obtained a Pieri-type rule for multiplying a general Schubert class by the Chern classes of the tautological quotient bundle on symplectic and orthogonal Grassmannians (to appear).

\section{References}

[AC] E. Akyildiz and J. B. Carrell, A generalization of the Kostant-Macdonald identity, Proc. Natl. Acad. Sci. USA 86 (1989), 3934-3937.

[Bo] A. Borel, Sur la cohomologie des espaces fibrés principaux et des espaces homogènes de groupes de Lie compacts, Ann. of Math. 57 (1953), 115-207.

[BGG] I. M. Bernstein, I. M. Gel'fand and S. I. Gel'fand, Schubert cells and cohomology of the spaces $G / P$, Russian Math. Surveys 28 (1973), no. 3, 1-26.

[Bou] N. Bourbaki, Groupes et Algèbres de Lie, Chapters 4, 5 and 6, Hermann, Paris, 1968.

[BKT] A. Buch, A. Kresch and H. Tamvakis, Gromov-Witten invariants on Grassmannians, preprint, 2002.

[C] C. Chevalley, Sur les décompositions cellulaires des espaces G/B, in: Algebraic Groups and Their Generalizations: Classical Methods, Proc. Sympos. Pure Math. 56, Amer. Math. Soc., 1994, 1-23.

[D1] M. Demazure, Invariants symétriques entiers des groupes de Weyl et torsion, Invent. Math. 21 (1973), 287-301.

[D2] - Désingularisation des variétés de Schubert géneralisées, Ann. Sci. École Norm. Sup. 7 (1974), 53-88.

[DP] H. Duan and P. Pragacz, Divided differences of type D and the Grassmannian of complex structures, in: Quantum Groups and Algebraic Combinatorics, N. Jing (ed.), World Sci., 2003, to appear.

[Exx] Examples to even orthogonal Pieri, manuscript (10 pages) available on P. Pragacz's web-page: http://www.impan.gov.pl/〜 pragacz/download/pieri.ps

$[\mathrm{FH}] \quad$ W. Fulton and J. Harris, Representation Theory. A First Course, Springer, 1991.

[GH] P. Griffiths and J. Harris, Principles of Algebraic Geometry, Wiley, 1978.

$[\mathrm{H}] \quad H$. Hiller, Combinatorics and intersections of Schubert varieties, Comment. Math. Helv. 57 (1982), 41-59.

[HB] H. Hiller and B. Boe, Pieri formula for $\mathrm{SO}_{2 n+1} / \mathrm{U}_{n}$ and $\mathrm{Sp}_{n} / \mathrm{U}_{n}$, Adv. Math. 62 (1986), 49-67.

[KK] B. Kostant and S. Kumar, The nil Hecke ring and cohomology of $G / P$ for a Kac-Moody group G, ibid. 62 (1986), 187-237.

[LP1] A. Lascoux and P. Pragacz, Operator calculus for $\widetilde{Q}$-polynomials and Schubert polynomials, ibid. 140 (1998), 1-43.

[LP2] - - , Orthogonal divided differences and Schubert polynomials, $\widetilde{P}$-functions, and vertex operators, Michigan Math. J. 48 (2000), 417-441.

[LS1] A. Lascoux et M.-P. Schützenberger, Polynômes de Schubert, C. R. Acad. Sci. Paris 294 (1982), 447-450.

[LS2] - - - Structure de Hopf de l'anneau de cohomologie et de l'anneau de Grothendieck d'une variété de drapeaux, ibid. 295 (1982), 629-633.

[LS3] - - Décompositions dans l'algèbre des différences divisées, Discrete Math. 99 (1992), 165-179. 
[M] I. G. Macdonald, Symmetric Functions and Hall Polynomials, 2nd ed., Oxford Univ. Press, 1995.

[Pi] M. Pieri, Sul problema degli spazi secanti, Rend. Inst. Lombardo 2 (1893), 534556 and 27 (1894), 258-273.

[P1] P. Pragacz, Symmetric polynomials and divided differences in formulas of intersection theory, in: Parameter Spaces, Banach Center Publ. 36, Inst. Math., Polish Acad. Sci., 1996, 125-177.

[P2] -, manuscript in preparation.

[PR1] P. Pragacz and J. Ratajski, Pieri type formula for isotropic Grassmannians; the operator approach, Manuscripta Math. 79 (1993), 127-151.

[PR2] - - - A Pieri-type theorem for Lagrangian and odd orthogonal Grassmannians, J. Reine Angew. Math. 476 (1996), 143-189.

[PR3] - - - A Pieri-type theorem for even orthogonal Grassmannians, Max-Planck Inst. Math. Preprint 96-83.

[PR4] - - Formulas of Lagrangian and orthogonal degeneracy loci; $\widetilde{Q}$-polynomial approach, Compositio Math. 107 (1997), 11-87.

[Se] C. Segre, Studio sulle quadriche in uno spazio lineare ad un numero qualunque di dimensioni, Mem. Della Reale Academia delle Scienze di Torino (2) 36 (1883), $3-86$.

[S] S. Sertöz, A triple intersection theorem for the varieties $S O(n) / P_{d}$, Fund. Math. 142 (1993), 201-220.

[So1] F. Sottile, Pieri's rule for flag manifolds and Schubert polynomials, Ann. Inst. Fourier (Grenoble) 46 (1996), 89-110.

[So2] - Pieri's formula via explicit rational equivalence, Canad. Math. J. 49 (1997), 1281-1298.

[So3] - Pieri-type formulas for maximal isotropic Grassmannians via triple intersections, Colloq. Math. 82 (1999), 49-63.

[V] S. Veigneau, thèse, Université de Marne-la-Vallée, 1996.

Institute of Mathematics

Polish Academy of Sciences

Śniadeckich 8

P.O. Box 21

00-956 Warszawa 10, Poland

E-mail: pragacz@impan.gov.pl
ING Nationale-Nederlanden Polska S.A.

Ludna 2

00-406 Warszawa, Poland E-mail: Jan.Ratajski@ingnn.pl

Received 31 January 2003;

in revised form 15 May 2003 